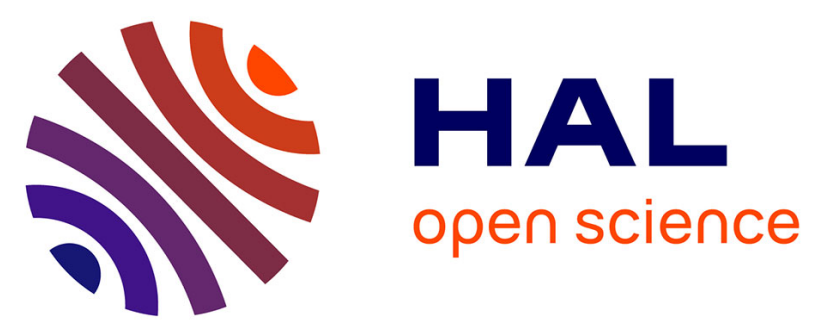

\title{
A Straightforward Access to Cyclotriveratrylene Analogues with C1-Symmetry: Toward the Synthesis of Monofunctionalizable Cryptophanes
}

Gaëlle Milanole, Grégory Pieters, Bo Gao, Emilie Mari, Bernard Rousseau, Patrick Berthault

\section{To cite this version:}

Gaëlle Milanole, Grégory Pieters, Bo Gao, Emilie Mari, Bernard Rousseau, et al.. A Straightforward Access to Cyclotriveratrylene Analogues with C1-Symmetry: Toward the Synthesis of Monofunctionalizable Cryptophanes. European Journal of Organic Chemistry, 2017, 47, pp.7091-7100. 10.1002/ejoc.201701353 . cea-01622050

\section{HAL Id: cea-01622050 https://hal-cea.archives-ouvertes.fr/cea-01622050}

Submitted on 7 Nov 2017

HAL is a multi-disciplinary open access archive for the deposit and dissemination of scientific research documents, whether they are published or not. The documents may come from teaching and research institutions in France or abroad, or from public or private research centers.
L'archive ouverte pluridisciplinaire HAL, est destinée au dépôt et à la diffusion de documents scientifiques de niveau recherche, publiés ou non, émanant des établissements d'enseignement et de recherche français ou étrangers, des laboratoires publics ou privés. 


\title{
A Straightforward Access to Cyclotriveratrylene Analogues with $C_{1}$-Symmetry: Toward the Synthesis of Monofunctionalizable Cryptophanes
}

\author{
Gaëlle Milanole, ${ }^{[a]}$ Bo Gao, ${ }^{[a]}$ Emilie Mari, ${ }^{[b]}$ Patrick Berthault, ${ }^{[b]}$ Grégory Pieters*, ${ }^{*}\left[{ }^{[a]}\right.$ and Bernard \\ Rousseau*,[a]
}

\begin{abstract}
A straightforward and practical access to various $C_{1-}$ symmetric cyclotriveratrylene derivatives from a monohalogenated cyclotriphenolene is described. This unique scaffold can also be used as a precursor for the rapid construction of monofunctionalizable cryptophane-based biosensors for hyperpolarized ${ }^{129} \mathrm{Xe} \mathrm{MRI.}$
\end{abstract}

\section{Introduction}

Cyclotriveratrylenes (CTVs) are versatile macrocyclic host molecules with a rigid bowl-shaped lipophilic and electron-rich cavity favoring the binding of guest molecules in a non-covalent way. ${ }^{[1]}$ In the last decades, CTVs and their analogues have received considerable interest in a wide range of applications such as selective fullerene separation, ${ }^{[2]}$ formation of liquid crystals $^{[3]}$ and organogels, ${ }^{[4]}$ anion sensing, ${ }^{[5]}$ metallosupramolecular assemblies ${ }^{[6]}$ and for their fluorescence properties. ${ }^{[7]}$

Since the early 1980 s, CTVs are also known as highly valuable precursors for the synthesis of cryptophanes. ${ }^{[8]}$ These cagemolecules made of two CTV units connected by alkylenedioxy linkers exhibit outstanding properties for xenon encapsulation. ${ }^{[9]}$ These host-guest assemblies have proven to be powerful systems for the hyperpolarized ${ }^{129} \mathrm{Xe}$ NMR-based biosensing approach, in which a biological ligand is grafted on a particular site of the cryptophane. ${ }^{[10]}$ Unfortunately, the high symmetry of these cryptophanes proved to be a drawback to control the reactivity of a particular position with respect to other equivalent sites: complex mixtures are reported, complicating the purification steps and preventing the production of large quantities of biosensor required for in vivo experiments. For this reason, the efficient introduction of a single chemical functionality on the aromatic rings of a symmetrical cryptophane needs to be addressed. Herein, we have focused our attention on the monofunctionalization of a CTV scaffold. To the best of our knowledge, only few $C_{1}$-symmetric CTV derivatives have been described in the literature. ${ }^{[11]}$ For example, one reported method involves a selective demethylation to furnish a single hydroxyl moiety with a low yield, then exploited to introduce a functional group (Scheme 1a). ${ }^{[11 b]}$

In this context, we describe here a new and alternative strategy to access novel $C_{1}$-symmetric CTV analogues based on the monohalogenation of cyclotri-phenolene 1 (Scheme 1b). These CTV backbones appear to be valuable precursors for designing monofunctionalized cryptophane-based biosensors.

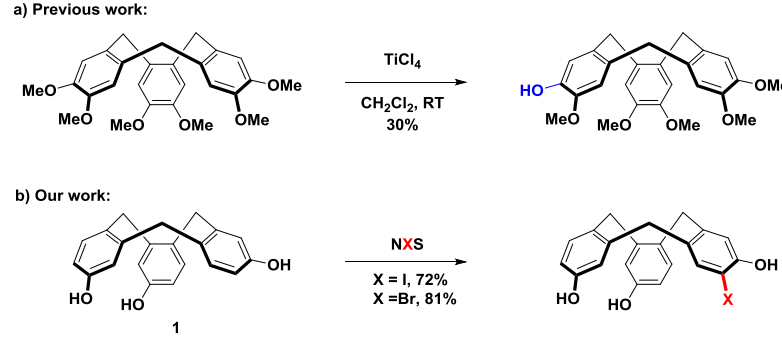

Scheme 1. A New Strategy to Access $C_{1}$-symmetric CTVs

\section{Results and Discussion}

Synthesis of $\boldsymbol{C}_{1}$-Symmetric CTV Derivatives. CTV 1 was obtained in two steps using a simple and scalable procedure elaborated by our group (40g-scale within 3 days). ${ }^{[12]}$ Monoiodation of compound $\mathbf{1}$ using $\mathrm{N}$-iodosuccinimide in acetonitrile gave rise to a mixture of mono-iodinated CTV 2 along with diiodinated CTV $\mathbf{3}$ and cyclotriphenolene $\mathbf{1}$ (Scheme 2a). Purification by chromatography on silica gel efficiently provided the expected mono-iodinated CTV 2 in a 72\% isolated yield (yield based on recovered starting material). It is noteworthy that unreacted cyclotriphenolene $\mathbf{1}$ was easily recovered. We were pleased to observe that the iodation takes place in a perfectly regioselective manner. Indeed, the ${ }^{1} \mathrm{H}$ NMR spectrum of CTV 2 unambiguously shows that iodination occurs at the ortho position of a phenoxy ring as outlined in Scheme $2 a$ (two singlets in the aromatic area at 7.74 and $7.00 \mathrm{ppm}$ ). Alternatively, we investigated the synthesis of mono-brominated scaffold 4 by a similar procedure using $\mathrm{N}$-bromosuccinimide (Scheme $2 b$ ). Surprisingly, the expected CTV $\mathbf{4}$ was obtained as a mixture of two regioisomers, easily separated on silica gel to afford $\mathbf{4 a}$ and $\mathbf{4 b}$ in $81 \%$ overall yield based on recovered starting material. Dibrominated CTV 5 was also observed. Most CTVs reported so far are substituted by electron-donating functions and only a few examples of CTVs substituted by electron-withdrawing groups or $\pi$-extended conjugated systems have been reported. ${ }^{[7 a, 13]}$ Indeed, CTV analogues are synthesized by an acid-catalyzed trimerization of 3,4-disubstituted benzyl alcohol compounds. The efficiency of this aromatic electrophilic substitution reaction $\left(\mathrm{SE}_{\mathrm{Ar}}\right)$ is mainly owed to the nature of 3-and 4-substituents which should be electron donors to strongly activate the aromatic ring. In our attempts to demonstrate the high potential of CTV 2 as a perfect key intermediate to extend the $C_{1}$-symmetric CTV family, we studied the introduction of electron-withdrawing or conjugated functions using either organolithium or cross-coupling chemistry. 
a)<smiles>Oc1ccc(CC2(c3ccc(O)cc3)Cc3ccc(O)cc3C2)cc1</smiles><smiles>Oc1ccc(CCc2ccc(O)cc2CCc2ccc(O)cc2)cc1</smiles>

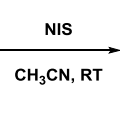

$\underset{\mathrm{CH}_{3} \mathrm{CN}, \mathrm{RT}}{\stackrel{\mathrm{NBS}}{\longrightarrow}}$

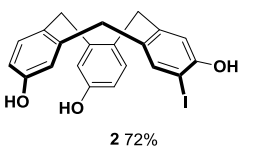

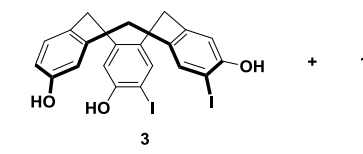<smiles>Oc1ccc(CCc2ccc(O)c(CCc3ccc(Br)c(O)c3)c2)cc1</smiles>
4a $81 \%$

Scheme 2. Synthesis of Monohalogenated CTV Derivatives

We decided to focus our study on iodinated derivative 2 for its expected higher reactivity in pallado-catalyzed reactions than the corresponding brominated compound $\mathbf{4 a}$. $^{[14]}$

First, CTV-phenylacetylene 6 was prepared through palladiumcatalyzed Sonogashira coupling of $\mathbf{2}$ with trimethylsilylacetylene (Scheme 3). Classical conditions using $\mathrm{PdCl}_{2}\left(\mathrm{PPh}_{3}\right)_{2}$ as a catalyst, $\mathrm{Cul}$ as a co-catalyst, excess of $\mathrm{Et}_{3} \mathrm{~N}$ and toluene as solvent, followed by a TBAF-mediated desilylation led to CTV 6 in $45 \%$ overall yield.<smiles>Oc1ccc(CCc2ccc(O)c(CCc3ccc(O)c(I)c3)c2)cc1</smiles>

2

$$
\begin{gathered}
\text { 1) TMS-acetylene } \\
\mathrm{PdCl}_{2}\left(\mathrm{PPh}_{3}\right)_{2}, \mathrm{Cul}^{-\mathrm{Et}_{3} \mathrm{~N}} \\
\text { toluene, } 40{ }^{\circ} \mathrm{C} \\
\hline \begin{array}{c}
45 \% \\
\text { 2) } \operatorname{TBAF}(1 \mathrm{M} \text { in THF), RT } \\
4
\end{array}
\end{gathered}
$$

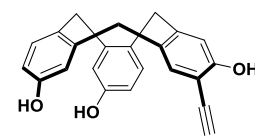

6

Scheme 3. Sonogashira Coupling from 2

We then turned our attention toward organolithium chemistry to introduce diverse electron-withdrawing groups. For that purpose, benzylation of phenolic moieties was initially carried out to protect the alcohol functionalities and to improve the solubility of monoiodinated CTV in THF. Treatment of CTV 2 with benzyl bromide, $\mathrm{K}_{2} \mathrm{CO}_{3}$ and catalytic amount of TBAI led efficiently to the protected CTV 7 in 92\% yield (Scheme 4).<smiles>Oc1ccc(CCc2ccc(O)cc2CCc2ccc(O)c(I)c2)cc1</smiles>

2

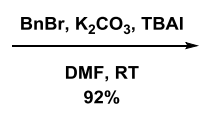
$92 \%$

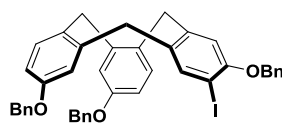

7

Scheme 4. Synthesis of Derivative 7

A halogen-metal exchange with $n$-BuLi at low temperature, followed by addition of ethyl chloroformate, converted CTV 7 to the corresponding ester 8 (Scheme 5). Then, removal of the benzyl protecting groups by hydrogenolysis over $\mathrm{Pd} / \mathrm{C}$ in EtOAc led to CTV 9 in $62 \%$ yield over two steps. Alternatively, reaction of the organolithium intermediate with DMF afforded the expected aldehyde 10 in $35 \%$ yield (Scheme 5).

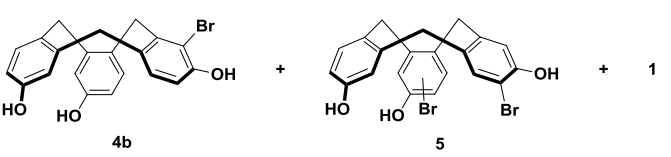

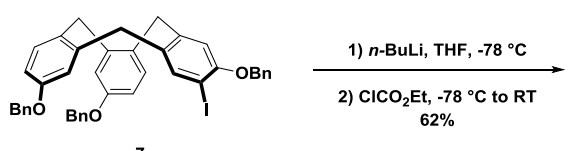

7 $62 \%$

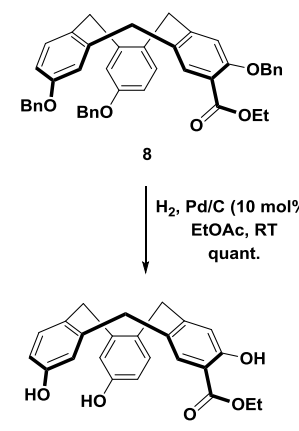

9

Scheme 5. Introduction of Electron-Withdrawing Groups using Organolithium Chemistry

Then, the reactivity of CTV 7 in classical conditions of SuzukiMiyaura coupling was explored as outlined in Scheme 6. Thus, cross-coupling with 4-methoxyphenylboronic acid using $\mathrm{Pd}(\mathrm{OAc})_{2}$ as catalyst, SPhos as ligand and anhydrous $\mathrm{K}_{3} \mathrm{PO}_{4}$ as base in refluxing toluene gave rise to CTV 11 in $64 \%$ yield (Scheme 6). The palladium-catalyzed Heck vinylation of iodinated CTV 7 was also investigated. Treatment with diethyl allylmalonate in the presence of $\mathrm{Pd}(\mathrm{OAc})_{2}, \mathrm{PPh}_{3}$ and $\mathrm{Et}_{3} \mathrm{~N}$ in refluxing DMF provided the $\pi$-extended conjugated CTV 12 in $50 \%$ yield (Scheme 6 ).

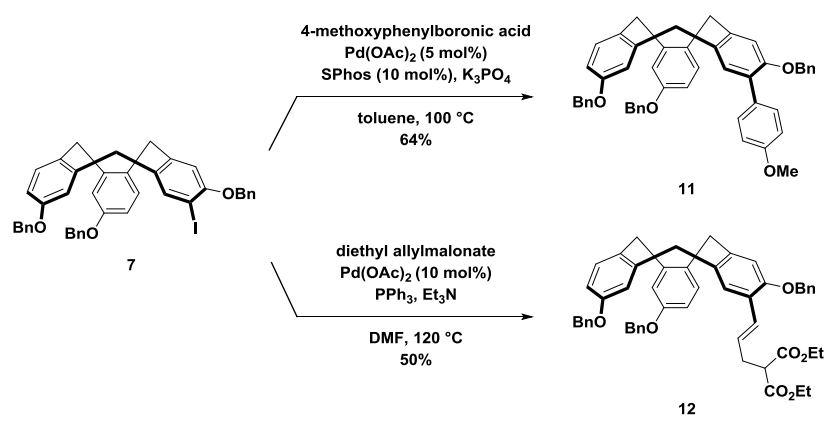

Scheme 6. Suzuki-Miyaura and Heck Cross-Coupling of 7

Finally, a Michaelis-Arbuzov reaction was successfully achieved using thiethylphosphite and nickel(II) chloride at $150{ }^{\circ} \mathrm{C}$, leading to the expected CTV 13 bearing a phosphonate moiety in $48 \%$ yield (Scheme 7).
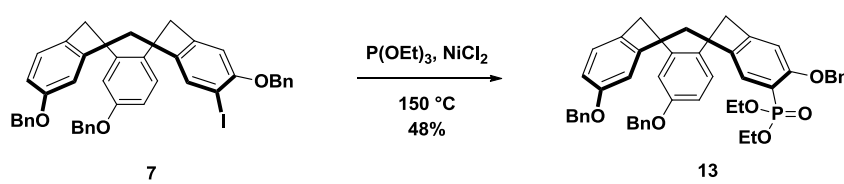

13

Scheme 7. Michaelis-Arbuzov Reaction from 7 
Synthesis of a Water-Soluble Monofunctionalizable Cryptophane. As part of our on-going research program dedicated to the efficient synthesis of a functionalizable watersoluble cryptophane, ${ }^{[15]}$ we decided to explore the potentiality of $C_{1}$-symmetric CTV 2 as a universal platform to access a wide range of original ${ }^{129} \mathrm{Xe} \mathrm{MRI}$-based biosensors bearing a single functionality. To date, the concept of cryptophane-xenon assemblies as ${ }^{129} \mathrm{Xe}$ NMR biosensing systems has not been extended to in vivo experiments owing to the limiting factor of scale. Indeed, a selective functionalization is required to introduce the recognition antenna of the biological target. Unfortunately, the high symmetry of cryptophane cores provides complex mixtures along with complicated purification steps, thus leading to unsatisfying yields and small quantities of biosensors. As a result, the construction of new cryptophane congeners from $C_{1-}$ symmetric CTVs described above is expected to provide two essential advantages: 1) diverse cryptophanes on a larger scale through the efficient monohalogenation of CTV $\mathbf{1}$ at the early stage of the synthetic strategy; 2) introduction of a specific biological antenna facilitated by taking advantage of the single functional group. Moreover, most biosensors described in the literature are based on cryptophanol-A. ${ }^{[10 \mathrm{~b}, \mathrm{f}, \mathrm{h}-\mathrm{m}]}$ However, the limited solubility of this hydrophobic cryptophane core in biological media results in the biosensor's uptake by biological membranes ${ }^{[16]}$ and formation of self-organized systems, ${ }^{[10 i]}$ hampering their use for in vitro or in vivo experiments. In this context, there is an urgent and unmet need to develop straightforward synthetic methods leading to simultaneously hydrophilic and $C_{1}$-symmetric cryptophane congeners. Recently, two water-soluble cryptophanes bearing a single functionality on their skeleton have been reported by Brotin et al. ${ }^{[17]}$ Herein, we describe the synthesis of one such cryptophane obtained from the $C_{1}$-symmetric CTV 2 and containing three poly(ethylene glycol) (PEG) chains as water-soluble moieties. To the best of our knowledge, this is the first time that introduction of a monofunctionality at the early stage of cryptophane synthesis is reported. The synthetic pathway started with the regioselective substitution of a phenol group of 3,4-dihydroxybenzaldehyde ${ }^{[18]}$ by a tosyl-functionalized PEG monomethyl ether $\mathbf{1 4}$ of eleven repeated units $\left(M_{n} 550\right)$ providing compound 15 in $84 \%$ yield (Scheme 8). An $\mathrm{S}_{\mathrm{N}} 2$ type reaction was performed in the presence of 1,2-dibromoethane and potassium carbonate to give rise to compound 16 in $56 \%$ yield. Reduction of the aldehyde function with $\mathrm{NaBH}_{4}$ at low temperature afforded the corresponding primary alcohol 17 in excellent $85 \%$ yield. Finally, protection of the alcohol function by a tetrahydropyranyl acetal led to compound 18 in $90 \%$ yield.

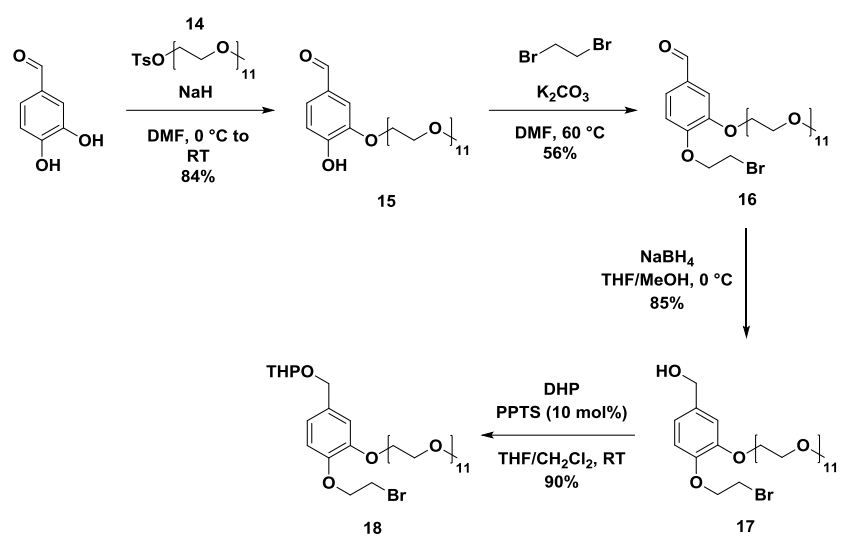

Scheme 8. Synthesis of compound 18

With pegylated linker $\mathbf{1 8}$ in hand, we applied the so-called template method to afford the expected cryptophane in two steps (Scheme 9). ${ }^{[8]}$ Deprotonation of CTV 2 with $\mathrm{Cs}_{2} \mathrm{CO}_{3}$, followed by treatment with compound $\mathbf{1 8}$ in DMF, led to the tris-functionalized intermediate 19 in $74 \%$ yield. Subsequent cyclotrimerization of this preorganized precursor with formic acid occurred affording the final compound $\mathbf{2 1}$ in a satisfying yield (41\%) after preparative HPLC purification.

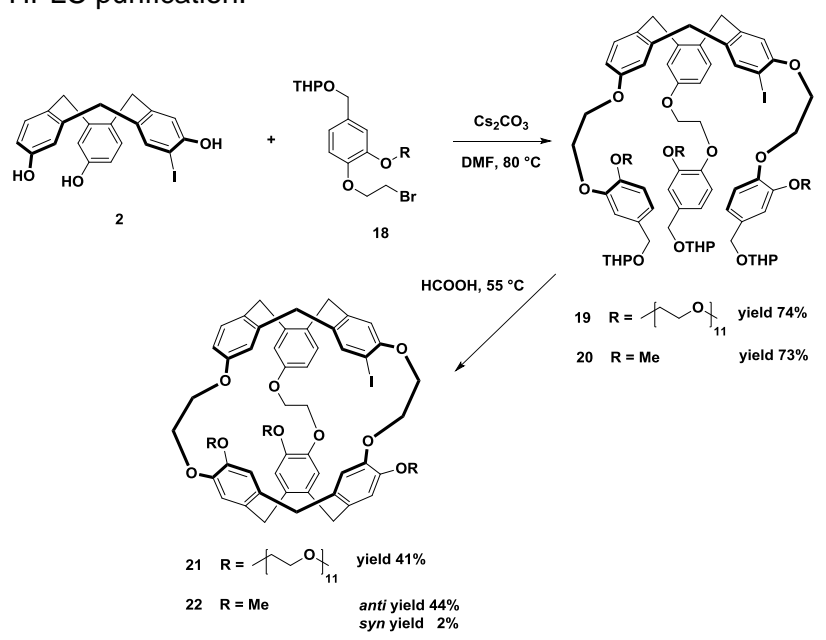

Scheme 9. Synthesis of Cryptophanes 21 and 22

NMR spectroscopic studies: In order to evaluate the xenon encapsulation properties of the new water-soluble cage, a purified sample of cryptophane $\mathbf{2 1}$ was then submitted to hyperpolarized ${ }^{129} \mathrm{Xe}$ NMR experiments. Two different ${ }^{129} \mathrm{Xe}$ NMR signals were observed (see ${ }^{129}$ Xe NMR spectra in SI), with chemical shifts of 60 and 75 ppm, attributed to the presence of anti and syn forms in the sample (difficult to separate because of the high polarity of the PEG chains). In order to confirm this hypothesis, the synthesis of cryptophane 22 was undertaken (Scheme 9). Syn and anti form were successfully separated and both forms showed satisfying encapsulation properties in tetrachloroethane, with xenon chemical shifts of 78 and $74 \mathrm{ppm}$, respectively (see ${ }^{129} \mathrm{Xe}$ NMR spectra in SI). Because of their ease of preparation and functionalization, we believe that the molecular cages described here can be considered as useful platforms for the construction of various cryptophane-based ${ }^{129} \mathrm{Xe} \mathrm{MRI}$ biosensors. 


\section{Conclusion}

In summary, we propose a new strategy for the straightforward synthesis of a monofunctionalized CTV from cyclotriphenolene. This $C_{1}$-symmetric compound is a perfect platform to add new members bearing electron-withdrawing or $\pi$-extended conjugated systems to the CTV family. Moreover, these valuable compounds open the way to a wide range of original cryptophanes for ${ }^{129} \mathrm{Xe}$ MRI biosensors. As an example, we describe a short and scalable synthetic route to a water-soluble $C_{1}$-symmetric cryptophane bearing an iodine atom. The single functionality on the aromatic scaffold is expected to allow the easy introduction of recognition antennas for various biosensing applications, avoiding the purification of complex statistical mixtures usually generated from $C_{3}$ or $D_{3}$-symmetric congeners.

\section{Experimental Section}

General Information. All reactions were conducted in oven-dried glassware under a nitrogen atmosphere. All moisture sensitive reactants were handled under a nitrogen atmosphere. Organic solvents were used without further purification. THF was distilled over sodium/benzophenone under a nitrogen atmosphere and $\mathrm{CH}_{2} \mathrm{Cl}_{2}$ over $\mathrm{CaH}_{2}$. Thin-layer chromatography (TLC) were performed on Merck 60 F254 silica gel plates, using UV light as a visualizing agent and ethanolic solution of phosphomolybdic acid and heat as developing agents. Flash column chromatography purifications were carried out by silica gel columns (particules size: $0.040-0.063 \mathrm{~mm}$ ) or by Combiflash $\AA$ chromatography on a RediSep apparatus using "pre-packed" silica cartridges. Infrared spectra (IR) were recorded on a Perkin Elmer system 2000 FT-IR spectrophotometer. Absorption bands are reported as wavelength numbers $\left(\mathrm{cm}^{-1}\right) .{ }^{1} \mathrm{H}$ NMR $(400.13 \mathrm{MHz}),{ }^{13} \mathrm{C}$ NMR $(100.62 \mathrm{MHz})$ spectra were recorded on a Bruker Avance $400 \mathrm{MHz}$ spectrometer. Chemical shifts are reported in parts per million (ppm, d) downfield from residual solvent peaks and coupling constants are reported as Hertz $(\mathrm{Hz})$. Splitting patterns are designated as singlet (s), doublet (d), triplet (t) or broad singulet (bs). Splitting patterns that could not be interpreted or easily visualized are designated as multiplet $(\mathrm{m}) .{ }^{129} \mathrm{Xe}$ NMR spectra were recorded on a Bruker Avance II $500 \mathrm{MHz}$ equipped with 5 $\mathrm{mm} \mathrm{HNX}$ and broadband inverse probeheads. Accurate calibration of the temperature was made using a methanol sample. Prior the introduction of the hyperpolarized noble gas, the solutions were degassed through helium bubbling or using several freeze-pump-thaw cycles. Electrospray mass spectra were recorded using an ESI/TOF Mariner Mass Spectrometer. High-resolution mass spectra (HRMS) were recorded on Waters LCT Premier. Unless otherwise noted, all other commercially available reagents were used without further purification.

Compound (2): In a $250 \mathrm{~mL}$ round-bottom flask equipped with a septum and a magnetic stirrer under nitrogen atmosphere, cyclotriphenolene 1 (300 mg, $0.942 \mathrm{mmol}$ ) was dissolved in $\mathrm{CH}_{3} \mathrm{CN}$ (100 mL). N-iodosuccinimide (212 mg, $0.942 \mathrm{mmol}$ ) was added and the reaction mixture was stirred for $30 \mathrm{~min}$ at room temperature. Then, the orange solution was quenched with water and concentrated in vacuo. The resulting residue was dissolved in EtOAc $(50 \mathrm{~mL})$, washed with water $(20 \mathrm{~mL})$ and the organic layer was separated. The aqueous layer was extracted with EtOAc $(3 \times 15 \mathrm{~mL})$, the combined organic layers were washed with brine, dried over anhydrous $\mathrm{MgSO}_{4}$ and concentrated in vacuo to afford a brownish solid. The resulting crude product was purified by column chromatography on silica gel (cyclohexane/EtOAc) to afford 2 as a pale yellow solid (184 mg, 72\%, yield based on recovered starting material). $\mathrm{R}_{f} 0.22$ (Cyclohexane/EtOAc: 6/4); MS (ESI positive mode): $\mathrm{m} / \mathrm{z} 462.0\left[\mathrm{M}+\mathrm{NH}_{4}\right]^{+}$; MS (ESI negative mode): m/z 443.1 [M-H]', 887.2 [2M-H]; IR (neat): 3286, 1687, 1606, 1585, 1496, 1476, 1443, 1395, 1233, 1147, 964, 867, 741, 633, $491 \mathrm{~cm}^{-1}$; HRMS (ESI positive mode) calcd. for $\mathrm{C}_{21} \mathrm{H}_{18} \mathrm{IO}_{3}$ : 445.029519; found: 445.029435; ${ }^{1} \mathrm{H}$ NMR $(400.13 \mathrm{MHz}$, acetone$\mathrm{d}_{6}$ ): $\delta 8.68$ (bs, $\left.1 \mathrm{H}\right), 8.10$ (bs, $\left.1 \mathrm{H}\right), 8.02$ (bs, $\left.1 \mathrm{H}\right), 7.74(\mathrm{~s}, 1 \mathrm{H}), 7.22$ $(\mathrm{d}, J=8.4 \mathrm{~Hz}, 1 \mathrm{H}), 7.16(\mathrm{~d}, J=8.4 \mathrm{~Hz}, 1 \mathrm{H}), 7.00(\mathrm{~s}, 1 \mathrm{H}), 6.92(\mathrm{~d}$, $J=2.6 \mathrm{~Hz}, 1 \mathrm{H}), 6.88(\mathrm{~d}, J=2.4 \mathrm{~Hz}, 1 \mathrm{H}), 6.58(\mathrm{dd}, J=2.6 \mathrm{~Hz}, J=$ $8.2 \mathrm{~Hz}, 1 \mathrm{H}), 6.56$ (dd, $J=2.6 \mathrm{~Hz}, J=8.2 \mathrm{~Hz}, 1 \mathrm{H}), 4.74$ (d, $J=13.3$ $\mathrm{Hz}, 3 \mathrm{H}), 3.55(\mathrm{~m}, 3 \mathrm{H}) ;{ }^{13} \mathrm{C}$ NMR (100.62 MHz, acetone- $\left.\mathrm{d}_{6}\right): \delta$ $156.9,155.9,143.3,142.6,141.9,141.0,134.3,132.0,131.7$, 131.6, 131.0, 117.2, 117.0, 114.8, 114.6, 82.0, 36.7, 36.4, 36.1.

Compounds (4a) and (4b): In a $250 \mathrm{~mL}$ round-bottom flask equipped with a septum and a magnetic stirrer under nitrogen atmosphere, cyclotriphenolene $1(100 \mathrm{mg}, 0.314 \mathrm{mmol})$ was dissolved in $\mathrm{CH}_{3} \mathrm{CN}$ (35 mL). $\mathrm{N}$-bromosuccinimide ( $56 \mathrm{mg}, 0.942$ $\mathrm{mmol}$ ) was added and the reaction mixture was stirred for $30 \mathrm{~min}$ at room temperature. Then, the orange solution was quenched with water and concentrated in vacuo. The resulting residue was dissolved in EtOAc $(30 \mathrm{~mL})$, washed with water $(15 \mathrm{~mL})$ and the organic layer was separated. The aqueous layer was extracted with $\mathrm{EtOAc}(3 \times 10 \mathrm{~mL})$, the combined organic layers were washed with brine, dried over anhydrous $\mathrm{MgSO}_{4}$ and concentrated in vacuo to afford a brownish solid. The resulting crude product was purified by column chromatography on silica gel (cyclohexane/EtOAc) to afford $\mathbf{4 a}$ and $\mathbf{4 b}$ as white solids (4a: 41.2 $\mathrm{mg}, 45 \%$; $4 \mathbf{b}$ : $32.4 \mathrm{mg}, 36 \%$, yields based on recovered starting material).

Isomer 4a: $\mathrm{R}_{f} 0.42$ (Cyclohexane/EtOAc: 1/1); MS (ESI positive mode): $\mathrm{m} / \mathrm{z} 414.0\left[\mathrm{M}+\mathrm{NH}_{4}\right]^{+}$; MS (ESI negative mode): $\mathrm{m} / \mathrm{z} 397.1$ [M-H]', 793.4 [2M-H]; IR (neat): 3295, 2989, 2916, 1686, 1604, 1496, 1476, 1439, 1400, 1227, 1146, 1108, 1089, 964, 868, 798, $742,634 \mathrm{~cm}^{-1}$; HRMS (ESI positive mode) calcd. for $\mathrm{C}_{21} \mathrm{H}_{18} \mathrm{BrO}_{3}$ : 397.043383; found: 397.043203; ${ }^{1} \mathrm{H}$ NMR $(400.13 \mathrm{MHz}$, acetone$\left.\mathrm{d}_{6}\right)$ : $\delta 7.53(\mathrm{~s}, 1 \mathrm{H}), 7.23(\mathrm{~d}, J=8.2 \mathrm{~Hz}, 1 \mathrm{H}), 7.18(\mathrm{~d}, J=8.2 \mathrm{~Hz}$, $1 \mathrm{H}), 7.05(\mathrm{~s}, 1 \mathrm{H}), 6.92(\mathrm{~d}, J=2.6 \mathrm{~Hz}, 1 \mathrm{H}), 6.89(\mathrm{~d}, J=2.6 \mathrm{~Hz}$, $1 \mathrm{H}), 6.58(\mathrm{dd}, J=2.6 \mathrm{~Hz}, J=8.4 \mathrm{~Hz}, 1 \mathrm{H}), 6.55(\mathrm{dd}, J=2.6 \mathrm{~Hz}, J$ $=8.2 \mathrm{~Hz}, 1 \mathrm{H}), 4.76(\mathrm{~m}, 3 \mathrm{H}), 3.57(\mathrm{~m}, 3 \mathrm{H}) ;{ }^{13} \mathrm{C}$ NMR $(100.62 \mathrm{MHz}$, acetone- $\left.\mathrm{d}_{6}\right): \delta 156.9,153.2,142.6,142.4,141.9,134.8,133.8$, $132.1,131.8,131.6,131.0,118.4,117.2,117.0,114.8,114.6$, $108.1,36.7,36.3,36.2$.

Isomer 4b: $\mathrm{R}_{f} 0.38$ (Cyclohexane/EtOAc: 1/1); MS (ESI positive mode): $\mathrm{m} / \mathrm{z} 398.9[\mathrm{M}+\mathrm{H}]^{+}$; MS (ESI negative mode): $\mathrm{m} / \mathrm{z} 396.1$ [MH]; IR (neat): 3254, 1689, 1585, 1497, 1470, 1438, 1235, 1148, 966, 867, 745, 711, 632, 555, $492 \mathrm{~cm}^{-1}$; HRMS (ESI positive mode) calcd. for $\mathrm{C}_{21} \mathrm{H}_{18} \mathrm{BrO}_{3}$ : 397.043383; found: 397.042726; ${ }^{1} \mathrm{H}$ 
NMR (400.13 MHz, acetone- $\left.\mathrm{d}_{6}\right): \delta 8,53$ (bs, 1H), 8.08 (s, 1H), 8.07 (s, $1 \mathrm{H}), 7.98(\mathrm{~d}, J=8.4 \mathrm{~Hz}, 1 \mathrm{H}), 7.33(\mathrm{~d}, J=8.2 \mathrm{~Hz}, 1 \mathrm{H}), 7.23(\mathrm{~d}$, $J=8.4 \mathrm{~Hz}, 1 \mathrm{H}), 6.95(\mathrm{~d}, J=2.6 \mathrm{~Hz}, 1 \mathrm{H}), 6.87(\mathrm{~d}, J=2.6 \mathrm{~Hz}, 1 \mathrm{H})$, $6.79(\mathrm{~d}, J=8.4 \mathrm{~Hz}, 1 \mathrm{H}), 6.59(\mathrm{dd}, J=2.6 \mathrm{~Hz}, J=8.2 \mathrm{~Hz}, 1 \mathrm{H}), 6.51$ (dd, $J=2.7 \mathrm{~Hz}, J=8.6 \mathrm{~Hz}, 1 \mathrm{H}), 4.82(\mathrm{~m}, 3 \mathrm{H}), 4.38(\mathrm{~d}, J=14.2$ $\mathrm{Hz}, 1 \mathrm{H}), 3.65$ (d, $J=13.7 \mathrm{~Hz}, 1 \mathrm{H}), 3.55(\mathrm{~d}, J=13.5 \mathrm{~Hz}, 1 \mathrm{H}) ;{ }^{13} \mathrm{C}$ NMR (100.62 MHz, acetone- $\left.d_{6}\right)$ : $\delta 156.9,153.8,143.7,142.0$, $141.3,134.1,132.6,132.4,131.6,131.5,130.6,117.4,117.2$, 115.2, 114.9, 114.1, 38.0, 37.1, 34.3.

Compound (6): In a $10 \mathrm{~mL}$ round-bottom flask equipped with a septum and a magnetic stirrer under nitrogen atmosphere, 2 (40 $\mathrm{mg}, 0.090 \mathrm{mmol})$, copper iodide $(3.4 \mathrm{mg}, 0.018 \mathrm{mmol})$ and bis(triphenylphosphine)palladium(II) dichloride (12.6 mg, 0.018 $\mathrm{mmol})$ were dissolved in toluene $(2.5 \mathrm{~mL})$. Subsequently, anhydrous triethylamine $(628 \mu \mathrm{L}, 4.50 \mathrm{mmol})$ and trimethylsilylacetylene ( $38 \mu \mathrm{L}, 0.270 \mathrm{mmol}$ ) were added. The reaction mixture was stirred for 24 hours at $40^{\circ} \mathrm{C}$. The reaction was cooled to room temperature and concentrated in vacuo. The brown crude oil was purified by column chromatography on silica gel (cyclohexane/EtOAc) to afford 3-((trimethylsilyl)ethynyl)10,15-dihydro-5H-tribenzo[a,d,g][9]annulene-2,7,12-triol as a pale yellow solid $(23.5 \mathrm{mg})$. Then, this intermediate was dissolved in THF (2 mL) under nitrogen atmosphere, and a $1 \mathrm{M}$ TBAF solution in THF $(0.198 \mathrm{~mL}, 0.198 \mathrm{mmol})$ was added slowly. The resulting solution was stirred at room temperature overnight until complete disappearance of starting material. The reaction mixture was dissolved in EtOAc $(15 \mathrm{~mL})$ and $\mathrm{H}_{2} \mathrm{O}(5 \mathrm{~mL})$ and the organic layer was separated. The aqueous layer was extracted with EtOAc $(3 \times 10 \mathrm{~mL})$, the combined organic layers were washed with brine, dried over anhydrous $\mathrm{MgSO}_{4}$ and concentrated in vacuo to afford 6 as a yellowish solid ( $18.2 \mathrm{mg}, 45 \%$ over two steps). $\mathrm{R}_{f}$ 0.36 (Cyclohexane/EtOAc: 1/1); MS (ESI positive mode): $\mathrm{m} / \mathrm{z}$ $343.1[\mathrm{M}+\mathrm{H}]^{+}, 685.4[2 \mathrm{M}+\mathrm{H}]^{+}$; MS (ESI negative mode): $\mathrm{m} / \mathrm{z} 341.2$ [M-H]; IR (neat): 3272, 2921, 2853, 1693, 1606, 1494, 1444, 1230, 1194, 1148, 1090, 965, 868, $743 \mathrm{~cm}^{-1}$; HRMS (ESI positive mode) calcd. for $\mathrm{C}_{23} \mathrm{H}_{19} \mathrm{O}_{3}$ : 343.132871; found: $343.133002 ;{ }^{1} \mathrm{H}$ $\operatorname{NMR}\left(400.13 \mathrm{MHz}\right.$, acetone- $\left.\mathrm{d}_{6}\right): \delta 8.17(\mathrm{~s}, 1 \mathrm{H}), 8.06(\mathrm{~s}, 1 \mathrm{H}), 8.02$ $(\mathrm{s}, 1 \mathrm{H}), 7.41(\mathrm{~s}, 1 \mathrm{H}), 7.22(\mathrm{~d}, J=8.4 \mathrm{~Hz}, 1 \mathrm{H}), 7.19(\mathrm{~d}, J=8.4 \mathrm{~Hz}$, $1 \mathrm{H}), 6.98(\mathrm{~s}, 1 \mathrm{H}), 6.91(\mathrm{~d}, J=2.6 \mathrm{~Hz}, 1 \mathrm{H}), 6.88(\mathrm{~d}, J=2.6 \mathrm{~Hz}$, $1 \mathrm{H}), 6.58-6.54(\mathrm{~m}, 2 \mathrm{H}), 4.77(\mathrm{~m}, 3 \mathrm{H}), 3.69(\mathrm{~s}, 1 \mathrm{H}), 3.56(\mathrm{~m}, 3 \mathrm{H})$; ${ }^{13} \mathrm{C}$ NMR $\left(100.62 \mathrm{MHz}\right.$, acetone- $\left.\mathrm{d}_{6}\right)$ : $\delta$ 158.0, 157.0, 144.3, 142.7, $142.0,135.6,132.3,132.0,131.9,131.6,130.9,117.5,117.3$, $117.1,114.8,114.7,108.7,83.0,80.7,36.8,36.7,36.4$.

Compound (7): In a $100 \mathrm{~mL}$ round-bottom flask equipped with a septum and a magnetic stirrer under nitrogen atmosphere, 2 (280 $\mathrm{mg}, 0.630 \mathrm{mmol})$, potassium carbonate $(1.74 \mathrm{~g}, 12.60 \mathrm{mmol})$ and tetrabutylammonium iodide $(69.8 \mathrm{mg}, 0.189 \mathrm{mmol})$ were dissolved in DMF $(55 \mathrm{~mL})$. Subsequently, benzyl bromide (1.48 $\mathrm{mL}, 12.60 \mathrm{mmol}$ ) was added and the reaction mixture was stirred overnight at room temperature until complete disappearance of starting material (monitored by TLC). The pale yellow suspension was diluted with EtOAc $(70 \mathrm{~mL})$ and water $(30 \mathrm{~mL})$. The aqueous layer was separated and extracted with EtOAc $(3 \times 20 \mathrm{~mL})$. The combined organic layers were washed with brine, dried over anhydrous $\mathrm{MgSO}_{4}$ and concentrated in vacuo. The resulting yellow crude oil was purified by column chromatography on silica gel (cyclohexane/EtOAc) to afford 7 as a white solid $(414.2 \mathrm{mg}$,
92\%). $\mathrm{R}_{f} 0.65$ (Cyclohexane/EtOAc: 8/2); MS (ESI positive mode): $\mathrm{m} / \mathrm{z} 731.9\left[\mathrm{M}+\mathrm{NH}_{4}\right]^{+}$; IR (neat): 3059, 3028, 2986, 2908, 2887, 2851, 1607, 1497, 1375, 1254, 1231, 1026, 731, $694 \mathrm{~cm}^{-1}$; HRMS (ESI positive mode) calcd. for $\mathrm{C}_{42} \mathrm{H}_{36} \mathrm{IO}_{3}: 715.170369$; found: 715.170512; ${ }^{1} \mathrm{H}$ NMR $\left(400.13 \mathrm{MHz}, \mathrm{CDCl}_{3}\right): \delta 7.76$ (s, $\left.1 \mathrm{H}\right), 7.50$ $7.33(\mathrm{~m}, 15 \mathrm{H}), 7.21(\mathrm{~d}, J=8.6 \mathrm{~Hz}, 1 \mathrm{H}), 7.00-6.97(\mathrm{~m}, 2 \mathrm{H}), 6.95$ $(\mathrm{d}, J=2.6 \mathrm{~Hz}, 1 \mathrm{H}), 6.78(\mathrm{~s}, 1 \mathrm{H}), 6.74(\mathrm{dd}, J=2.6 \mathrm{~Hz}, J=8.6 \mathrm{~Hz}$, $1 \mathrm{H}), 6.64(\mathrm{dd}, J=2.6 \mathrm{~Hz}, J=8.6 \mathrm{~Hz}, 1 \mathrm{H}), 5.22-5.02(\mathrm{~m}, 6 \mathrm{H}), 4.69$ $(\mathrm{m}, 3 \mathrm{H}), 3.58(\mathrm{~m}, 3 \mathrm{H}) ;{ }^{13} \mathrm{C}$ NMR $\left(100.62 \mathrm{MHz}, \mathrm{CDCl}_{3}\right): \delta 157.6$, $157.5,155.8,141.3,140.8,140.5,140.3,137.1,137.0,136.7$, 133.6, 131.8, 131.1, 130.6, 128.6, 128.5, 128.0, 127.8, 127.7, $127.5,127.3,126.8,116.4,116.2,114.3,113.2,113.1,84.5,71.0$, $70.1,69.9,36.4,36.1$.

Compound (8): In a $25 \mathrm{~mL}$ round-bottom flask equipped with a septum and a magnetic stirrer under nitrogen atmosphere, 7 (20 $\mathrm{mg}, 0.028 \mathrm{mmol})$ was dissolved in THF $(4.25 \mathrm{~mL})$. The reaction mixture was cooled to $-78^{\circ} \mathrm{C}$ and $n$-BuLi $(2.5 \mathrm{M}$ in THF, $56 \mu \mathrm{L}$, $0.140 \mathrm{mmol}$ ) was added dropwise. The resulting yellow solution was stirred at $-78{ }^{\circ} \mathrm{C}$ for $15 \mathrm{~min}$. Then, freshly distilled ethyl chloroformate $(80 \mu \mathrm{L}, 0.840 \mathrm{mmol})$ was added and the mixture was stirred at $-78{ }^{\circ} \mathrm{C}$ for 2 hours and at room temperature for 2 hours. Upon completion, the pale yellow solution was quenched with a saturated $\mathrm{NH}_{4} \mathrm{Cl}$ aqueous solution at $0{ }^{\circ} \mathrm{C}$. The mixture was diluted with EtOAc and the organic layer was separated. The aqueous layer was extracted with EtOAc $(3 \times 10 \mathrm{~mL})$. The combined organic layers were washed with brine, dried over anhydrous $\mathrm{MgSO}_{4}$ and concentrated in vacuo. The crude oil was purified by column chromatography on silica gel (cyclohexane/EtOAc) to afford 8 as a white solid $(11.3 \mathrm{mg}, 62 \%)$. Rf 0.35 (Cyclohexane/EtOAc: 8/2); MS (ESI positive mode): $\mathrm{m} / \mathrm{z}$ $661.5[\mathrm{M}+\mathrm{H}]+, 1339.2\left[2 \mathrm{M}+\mathrm{NH}_{4}\right]^{+}$; IR (neat): 3033, 2963, 2921, 2870, 1699, 1606, 1496, 1453, 1410, 1378, 1248, 1148, 1027, 729, $693 \mathrm{~cm}^{-1}$; HRMS (ESI positive mode) calcd. for $\mathrm{C}_{45} \mathrm{H}_{41} \mathrm{O}_{5}$ : 661.294851; found: $661.295026 ;{ }^{1} \mathrm{H}$ NMR $\left(400.13 \mathrm{MHz}, \mathrm{CDCl}_{3}\right)$ : $\delta 7.85(\mathrm{~s}, 1 \mathrm{H}), 7.49-7.30(\mathrm{~m}, 15 \mathrm{H}), 7.19(\mathrm{~d}, J=8.6 \mathrm{~Hz}, 1 \mathrm{H}), 7.03$ $(\mathrm{d}, J=2.6 \mathrm{~Hz}, 1 \mathrm{H}), 6.98(\mathrm{~d}, J=8.6 \mathrm{~Hz}, 1 \mathrm{H}), 6.94(\mathrm{~d}, J=2.6 \mathrm{~Hz}$, $1 \mathrm{H}), 6.93(\mathrm{~s}, 1 \mathrm{H}), 6.72(\mathrm{dd}, J=2.6 \mathrm{~Hz}, J=8.4 \mathrm{~Hz}, 1 \mathrm{H}), 6.63$ (dd, $J=2.7 \mathrm{~Hz}, J=8.6 \mathrm{~Hz}, 1 \mathrm{H}), 5.25-5.02(\mathrm{~m}, 6 \mathrm{H}), 4.75(\mathrm{~m}, 3 \mathrm{H}), 4.35$ (q, $J=7.0 \mathrm{~Hz}, 2 \mathrm{H}), 3.65(\mathrm{~m}, 3 \mathrm{H}), 1.34(\mathrm{t}, J=7.1 \mathrm{~Hz}, 3 \mathrm{H}) ;{ }^{13} \mathrm{C}$ NMR $\left(100.62 \mathrm{MHz}, \mathrm{CDCl}_{3}\right): \delta 166.2,157.7,157.6,156.7,145.5,141.1$ $140.4,137.0,133.6,131.8,131.4,131.1,130.8,130.7,128.6$, $128.5,128.0,127.9,127.7,127.6,127.4,126.9,119.6,116.3$, $116.2,115.6,113.3,113.2,70.9,70.0,60.8,36.7,36.5,36.4$, 14.3.

Compound (9): A solution of $8(11.2 \mathrm{mg}, 0.017 \mathrm{mmol})$ and palladium (10\% wt. on carbon, $1.8 \mathrm{mg}, 0.002 \mathrm{mmol}$ ) in EtOAc (2 $\mathrm{mL}$ ) was stirred under an atmospheric pressure of hydrogen at room temperature for 16 hours, until complete disappearance of starting material (monitored by TLC). Upon completion, the reaction mixture was filtered through a pad of Celite $\AA$ and the filtrate was concentrated in vacuo to afford $9(6.5 \mathrm{mg}$, quantitative). No further purification was needed. $\mathrm{R}_{f} 0.58$ (Cyclohexane/EtOAc: 1/1); MS (ESI positive mode): $\mathrm{m} / \mathrm{z} 391.2$ $[\mathrm{M}+\mathrm{H}]^{+}, 408.3\left[\mathrm{M}+\mathrm{NH}_{4}\right]^{+}, 798.6\left[2 \mathrm{M}+\mathrm{NH}_{4}\right]^{+} ; \mathrm{MS}$ (ESI negative mode): m/z 389.3 [M-H]', 779.6 [2M-H]; IR (neat): 3380, 3355, 2920, 2851, 1674, 1618, 1582, 1493, 1463, 1376, 1263, 1213 1147, 788, 741, $492 \mathrm{~cm}^{-1}$; HRMS (ESI positive mode) calcd. for 
$\mathrm{C}_{24} \mathrm{H}_{23} \mathrm{O}_{5}$ : 391.154000; found: 391.154017; ${ }^{1} \mathrm{H}$ NMR (400.13 $\mathrm{MHz}$, acetone- $\left.\mathrm{d}_{6}\right): \delta 10.47(\mathrm{~s}, 1 \mathrm{H}), 8.09(\mathrm{~s}, 1 \mathrm{H}), 8.07(\mathrm{~s}, 1 \mathrm{H}), 7.90$ (s, $1 \mathrm{H}), 7.30(\mathrm{~d}, J=8.4 \mathrm{~Hz}, 1 \mathrm{H}), 7.23(\mathrm{~d}, J=8.4 \mathrm{~Hz}, 1 \mathrm{H}), 7.04(\mathrm{~s}$, $1 \mathrm{H}), 6.90(\mathrm{~d}, J=2.6 \mathrm{~Hz}, 1 \mathrm{H}), 6.88(\mathrm{~d}, J=2.5 \mathrm{~Hz}, 1 \mathrm{H}), 6.60(\mathrm{~d}, J$ $=1.1 \mathrm{~Hz}, 1 \mathrm{H}), 6.58(\mathrm{~d}, J=1.1 \mathrm{~Hz}, 1 \mathrm{H}), 4.81(\mathrm{~m}, 3 \mathrm{H}), 4.39(\mathrm{~m}, 2 \mathrm{H})$, $3.62(\mathrm{~m}, 3 \mathrm{H}), 1.39(\mathrm{t}, J=7.1 \mathrm{~Hz}, 3 \mathrm{H}) ;{ }^{13} \mathrm{C}$ NMR $(100.62 \mathrm{MHz}$, acetone- $\left.\mathrm{d}_{6}\right): \delta 170.7,160.9,157.1,157.0,150.5,142.8,141.8$, $132.3,132.2,132.1,131.8,130.2,119.0,117.2,117.1,114.9$, $114.8,111.9,62.2,36.9,36.8,36.6,14.6$.

Compound (10): In a $25 \mathrm{~mL}$ round-bottom flask equipped with a septum and a magnetic stirrer under nitrogen atmosphere, 7 (20 $\mathrm{mg}, 0.028 \mathrm{mmol}$ ) was dissolved in THF $(4.25 \mathrm{~mL})$. The reaction mixture was cooled to $-78^{\circ} \mathrm{C}$ and $n$-BuLi $(2.5 \mathrm{M}$ in THF, $56 \mu \mathrm{L}$, $0.140 \mathrm{mmol}$ ) was added dropwise. The resulting yellow solution was stirred at $-78{ }^{\circ} \mathrm{C}$ for $15 \mathrm{~min}$. Then, $\mathrm{N}, \mathrm{N}$-dimethylformamide $(65 \mu \mathrm{L}, 0.840 \mathrm{mmol})$ was added and the mixture was stirred at $78{ }^{\circ} \mathrm{C}$ for 2 hours and at room temperature for 2 hours. Upon completion, the pale yellow solution was quenched with a saturated $\mathrm{NH}_{4} \mathrm{Cl}$ aqueous solution at $0{ }^{\circ} \mathrm{C}$. The mixture was diluted with EtOAc and the organic layer was separated. The aqueous layer was extracted with EtOAc $(3 \times 10 \mathrm{~mL})$. The combined organic layers were washed with brine, dried over anhydrous $\mathrm{MgSO}_{4}$ and concentrated in vacuo. The crude oil was purified by column chromatography on silica gel (cyclohexane/EtOAc) to afford $\mathbf{1 0}$ as a white solid (6.0 mg, 35\%). $\mathrm{R}_{f} 0.55$ (Cyclohexane/EtOAc: $7 / 3$ ); MS (ESI positive mode): $\mathrm{m} / \mathrm{z}$ $617.5[\mathrm{M}+\mathrm{H}]^{+}, 1251.6\left[2 \mathrm{M}+\mathrm{NH}_{4}\right]^{+}$; MS (ESI negative mode): $\mathrm{m} / \mathrm{z}$ 615.5 [M-H]; IR (neat): 3058, 3026, 2919, 2855, 1672, 1603, 1494, 1453, 1248, 1025, 729, $692 \mathrm{~cm}^{-1}$; HRMS (ESI positive mode) calcd. for $\mathrm{C}_{43} \mathrm{H}_{37} \mathrm{O}_{4}$ : 617.268636; found: $617.268918 ;{ }^{1} \mathrm{H}$ $\operatorname{NMR}\left(400.13 \mathrm{MHz}, \mathrm{CDCl}_{3}\right)$ : $\delta 10.41(\mathrm{~s}, 1 \mathrm{H}), 7.84(\mathrm{~s}, 1 \mathrm{H}), 7.43-$ $7.35(\mathrm{~m}, 15 \mathrm{H}), 7.17(\mathrm{~d}, J=8.4 \mathrm{~Hz}, 1 \mathrm{H}), 7.02(\mathrm{~d}, J=2.6 \mathrm{~Hz}, 1 \mathrm{H})$, $7.00(\mathrm{~d}, J=8.4 \mathrm{~Hz}, 1 \mathrm{H}), 6.95(\mathrm{~s}, 1 \mathrm{H}), 6.94(\mathrm{~d}, J=2.6 \mathrm{~Hz}, 1 \mathrm{H})$, $6.70(\mathrm{dd}, J=2.7 \mathrm{~Hz}, J=8.4 \mathrm{~Hz}, 1 \mathrm{H}), 6.63(\mathrm{dd}, J=2.6 \mathrm{~Hz}, J=8.4$ $\mathrm{Hz}, 1 \mathrm{H}), 5.27-5.00(\mathrm{~m}, 6 \mathrm{H}), 4.74(\mathrm{~m}, 3 \mathrm{H}), 3.65(\mathrm{~m}, 3 \mathrm{H}) ;{ }^{13} \mathrm{C}$ NMR $\left(100.62 \mathrm{MHz}, \mathrm{CDCl}_{3}\right): \delta 189.1,159.5,157.7,157.6,148.4,141.2$, $140.1,137.0,136.9,136.3,132.1,131.6,131.0,130.7,130.1$, $128.8,128.5,128.1,127.9,127.5,127.3,127.1,123.9,116.3$, $116.0,114.5,113.6,113.3,70.5,70.1,70.0,36.9,36.6,36.3$.

Compound (11): In a $10 \mathrm{~mL}$ round-bottom flask equipped with a septum, a magnetic stirrer and a reflux condenser under nitrogen atmosphere, 7 (16.0 mg, $0.022 \mathrm{mmol})$, 4-methoxyphenylboronic acid $(6.8 \mathrm{mg}, 0.045 \mathrm{mmol})$, SPhos $(0.9 \mathrm{mg}, 2.25 \mu \mathrm{mol})$, palladium acetate $(0.25 \mathrm{mg}, 1.12 \mu \mathrm{mol})$ and anhydrous tripotassium phosphate $(14.3 \mathrm{mg}, 0.068 \mathrm{mmol})$ were dissolved in toluene $(1.0$ $\mathrm{mL})$. The reaction mixture was stirred overnight at $100{ }^{\circ} \mathrm{C}$ until complete disappearance of starting material (monitored by TLC). Subsequently, the mixture was allowed to cool to room temperature and concentrated in vacuo. The resulting brownish solid was purified by column chromatography on silica gel (cyclohexane/EtOAc) to afford $\mathbf{1 1}$ as a pale yellow solid $(10.0 \mathrm{mg}$, 64\%). R 0.31 (Cyclohexane/EtOAc: 9/1); IR (neat): 3027, 2919, 2853, 1608, 1492, 1452, 1377, 1250, 1027, 833, 730, $693 \mathrm{~cm}^{-1}$; HRMS (ESI positive mode) calcd. for $\mathrm{C}_{49} \mathrm{H}_{43} \mathrm{O}_{4}$ : 695.315867; found: $695.315586 ;{ }^{1} \mathrm{H}$ NMR $\left(400.13 \mathrm{MHz}, \mathrm{CDCl}_{3}\right): \delta 7.47$ (d, J = $8.4 \mathrm{~Hz}, 2 \mathrm{H}), 7.41-7.28(\mathrm{~m}, 16 \mathrm{H}), 7.21(\mathrm{~d}, J=8.4 \mathrm{~Hz}, 1 \mathrm{H}), 7.05$ (d, $J=8.4 \mathrm{~Hz}, 1 \mathrm{H}), 6.99(\mathrm{~d}, J=2.6 \mathrm{~Hz}, 1 \mathrm{H}), 6.96-6.93(\mathrm{~m}, 4 \mathrm{H}), 6.71$ (dd, $J=2.6 \mathrm{~Hz}, J=8.4 \mathrm{~Hz}, 1 \mathrm{H}), 6.65(\mathrm{dd}, J=2.6 \mathrm{~Hz}, J=8.4 \mathrm{~Hz}$, $1 \mathrm{H}), 5.12-5.00(\mathrm{~m}, 6 \mathrm{H}), 4.79(\mathrm{~d}, J=13.6 \mathrm{~Hz}, 3 \mathrm{H}), 3.87(\mathrm{~s}, 3 \mathrm{H})$, $3.65(\mathrm{~m}, 3 \mathrm{H}) ;{ }^{13} \mathrm{C}$ NMR $\left(100.62 \mathrm{MHz}, \mathrm{CDCl}_{3}\right): \delta 158.5,157.6$ $157.4,154.2$, 141.0, 140.9, 139.6, 137.5, 137.2, 137.1, 132.3, $131.9,131.7,131.6,131.0,130.8,130.8,129.6,128.6,128.5$, $128.4,127.9,127.5,127.4,126.8,116.5,116.2,114.9,113.4$, $113.2,113.0,70.6,70.1,70.0,55.3,36.7,36.5,36.4$.

Compound (12): In a $10 \mathrm{~mL}$ round-bottom flask equipped with a septum, a magnetic stirrer and a reflux condenser under nitrogen atmosphere, 7 (20.0 mg, $0.028 \mathrm{mmol})$, palladium acetate $(0.7 \mathrm{mg}$, $2.8 \mu \mathrm{mol})$ and triphenylphosphine $(14.7 \mathrm{mg}, 0.056 \mathrm{mmol})$ were dissolved in DMF. Then, triethylamine $(19 \mu \mathrm{L}, 0.140 \mathrm{mmol})$ was added and diethyl allylmalonate $(55 \mu \mathrm{L}, 0.280 \mathrm{mmol})$ was added slowly. The reaction mixture was stirred overnight under reflux until complete disappearance of starting material (monitored by TLC). Subsequently, the mixture was allowed to cool to room temperature and concentrated in vacuo. The resulting brownish solid was purified by column chromatography on silica gel (cyclohexane/EtOAc) to afford 11 as a white solid (11.2 mg, 50\%). $\mathrm{R}_{f} 0.35$ (Cyclohexane/EtOAc: 8/2); MS (ESI positive mode): $\mathrm{m} / \mathrm{z}$ $804.6\left[\mathrm{M}+\mathrm{NH}_{4}\right]^{+}$; IR (neat): 3030, 2986, 2920, 2862, 1727, 1605, 1496, 1453, 1248, 1149, 1025, 857, 734, $695 \mathrm{~cm}^{-1}$; HRMS (ESI positive mode) calcd. for $\mathrm{C}_{52} \mathrm{H}_{51} \mathrm{O}_{7}$ : 787.362930; found: 787.363256; ${ }^{1} \mathrm{H} \mathrm{NMR}\left(400.13 \mathrm{MHz}, \mathrm{CDCl}_{3}\right): \delta 7.45-7.31(\mathrm{~m}, 15 \mathrm{H})$, $7.17(\mathrm{~d}, J=8.4 \mathrm{~Hz}, 1 \mathrm{H}), 6.99(\mathrm{~m}, 2 \mathrm{H}), 6.92(\mathrm{~d}, J=2.6 \mathrm{~Hz}, 1 \mathrm{H})$, $6.80(\mathrm{~s}, 1 \mathrm{H}), 6.69(\mathrm{~m}, 2 \mathrm{H}), 6.62(\mathrm{dd}, J=2.6 \mathrm{~Hz}, J=8.4 \mathrm{~Hz}, 1 \mathrm{H})$, $6.13(\mathrm{~m}, 1 \mathrm{H}), 5.14-5.00(\mathrm{~m}, 6 \mathrm{H}), 4.72(\mathrm{~m}, 3 \mathrm{H}), 4.13(\mathrm{~m}, 4 \mathrm{H}), 3.60$ $(\mathrm{m}, 3 \mathrm{H}), 3.45(\mathrm{t}, J=7.5 \mathrm{~Hz}, 1 \mathrm{H}), 2.79(\mathrm{~m}, 2 \mathrm{H}), 1.20(2 \mathrm{t}, J=7.2$ $\mathrm{Hz}, 6 \mathrm{H}) ;{ }^{13} \mathrm{C}$ NMR $(100.62 \mathrm{MHz}, \mathrm{CDCl} 3): \delta 168.9,157.6,157.4$, $154.4,140.9,140.8,140.0,137.3,137.2,131.8,131.6,131.4$, $131.0,130.7,128.6,127.9,127.8,127.6,127.5,127.3,127.1$, $126.0,125.3,116.3,116.2,113.9,113.1,113.0,70.4,70.0,61.3$, $52.2,36.6,36.5,32.7,14.1$.

Compound (13): In a sealed tube equipped with a magnetic stirrer under nitrogen atmosphere, $7(10.0 \mathrm{mg}, 0.014 \mathrm{mmol})$ and anhydrous nickel chloride ( $3.6 \mathrm{mg}, 0.028 \mathrm{mmol}$ ) were dissolved in triethylphosphite $(0.5 \mathrm{~mL})$. The reaction mixture was stirred for 5 days at $150^{\circ} \mathrm{C}$ until complete disappearance of starting material. Subsequently, the mixture was allowed to cool to room temperature and concentrated in vacuo. The resulting yellow oil was purified by column chromatography on silica gel (cyclohexane/EtOAc) to afford 13 as a yellow solid (4.8 mg, 48\%). $\mathrm{R}_{f} 0.35$ (Cyclohexane/EtOAc: 1/1) ; MS (ESI positive mode): $\mathrm{m} / \mathrm{z}$ $725.6[\mathrm{M}+\mathrm{H}]^{+}, 1450.3[2 \mathrm{M}+\mathrm{H}]^{+}$; IR (neat): 3030, 2980, 2924, 1603, 1496, 1245, 1021, 966, 732, $695 \mathrm{~cm}^{-1}$; HRMS (ESI positive mode) calcd. for $\mathrm{C}_{46} \mathrm{H}_{46} \mathrm{O}_{6} \mathrm{P}: 725.302652$; found: 725.303277 ; ${ }^{1} \mathrm{H}$ NMR (400.13 MHz, $\left.\mathrm{CDCl}_{3}\right)$ : $\delta 7.88$ (d, $\left.J=15.2 \mathrm{~Hz}, 1 \mathrm{H}\right), 7.48-7.31(\mathrm{~m}$, $15 \mathrm{H}), 7.18(\mathrm{~d}, J=8.4 \mathrm{~Hz}, 1 \mathrm{H}), 7.04(\mathrm{~d}, J=1.8 \mathrm{~Hz}, 1 \mathrm{H}), 6.98(\mathrm{~d}, J$ $=8.4 \mathrm{~Hz}, 1 \mathrm{H}), 6.94(\mathrm{~d}, J=2.0 \mathrm{~Hz}, 1 \mathrm{H}), 6.87(\mathrm{~d}, J=6.6 \mathrm{~Hz}, 1 \mathrm{H})$, 6.70 (dd, $J=2.0 \mathrm{~Hz}, J=8.4 \mathrm{~Hz}, 1 \mathrm{H}), 6.62(\mathrm{dd}, J=2.1 \mathrm{~Hz}, J=8.2$ $\mathrm{Hz}, 1 \mathrm{H}), 5.26-5.00(\mathrm{~m}, 6 \mathrm{H}), 4.74(\mathrm{~m}, 3 \mathrm{H}), 4.08(\mathrm{~m}, 4 \mathrm{H}), 3.64(\mathrm{~m}$, $3 \mathrm{H}), 1.26(\mathrm{~m}, 6 \mathrm{H}) ;{ }^{13} \mathrm{C}$ NMR $\left(100.62 \mathrm{MHz}, \mathrm{CDCl}_{3}\right)$ : $\delta$ 157.7, 157.6, $146.2,141.2,140.4,137.0,136.7,131.6,131.0,130.7,130.5$, $128.6,128.5,127.9,127.8,127.7,127.4,127.3,126.8,116.2$, $116.0,113.5,113.2,70.2,69.9,63.6,63.5,36.8,36.5,36.3,16.2$, 16.1 
Compound (15): In a $25 \mathrm{~mL}$ round-bottom flask equipped with a septum and a magnetic stirrer under nitrogen atmosphere, sodium hydride $95 \%$ (225.0 $\mathrm{mg}, 9.39 \mathrm{mmol})$ was suspended in DMF $(2.5 \mathrm{~mL})$ and the resulting suspension was cooled to $0{ }^{\circ} \mathrm{C}$. Then, 3,4-dihydroxybenzaldehyde (618.3 mg, $4.47 \mathrm{mmol})$, previously dissolved in DMF $(2.5 \mathrm{~mL})$, was added dropwise and the yellow solution was stirred at $0{ }^{\circ} \mathrm{C}$ for $30 \mathrm{~min}$. Subsequently, $14(3.00 \mathrm{~g}, 4.47 \mathrm{mmol})$, previously dissolved in DMF $(2.5 \mathrm{~mL})$, was added slowly and the reaction mixture was stirred at room temperature overnight. Upon completion, the mixture was concentrated in vacuo, the residue was dissolved in $\mathrm{CH}_{2} \mathrm{Cl}_{2}(50$ $\mathrm{mL}$ ) and a $1 \mathrm{M} \mathrm{HCl}$ aqueous solution was added until $\mathrm{pH}$ 2. The aqueous layer was separated and extracted with $\mathrm{CH}_{2} \mathrm{Cl}_{2}(3 \times 20$ $\mathrm{mL}$ ). The combined organic layers were washed with brine, dried over anhydrous $\mathrm{MgSO}_{4}$ and concentrated in vacuo. The resulting brownish oil was purified by column chromatography on silica gel $\left(\mathrm{CH}_{2} \mathrm{Cl}_{2} / \mathrm{MeOH}\right)$ to afford 15 as a pale yellow oil $(2.40 \mathrm{~g}, 84 \%) . \mathrm{R}_{f}$ $0.55\left(\mathrm{CH}_{2} \mathrm{Cl}_{2} / \mathrm{MeOH}: 9 / 1\right)$; MS (ESI positive mode): $\mathrm{m} / \mathrm{z} 637.4$ $[\mathrm{M}+\mathrm{H}]^{+}, 654.4\left[\mathrm{M}+\mathrm{NH}_{4}\right]^{+} ; \mathrm{MS}$ (ESI negative mode): $\mathrm{m} / \mathrm{z} 635.5[\mathrm{M}-$ H]; IR (neat): 3504, 2870, 1682, 1588, 1512, 1442, 1349, 1292 , 1251, 1093, 948, 847, $633 \mathrm{~cm}^{-1}$; HRMS (ESI positive mode) calcd. for $\mathrm{C}_{30} \mathrm{H}_{53} \mathrm{O}_{14}$ : 637.342983; found: 637.343011; ${ }^{1} \mathrm{H}$ NMR $(400.13$ $\left.\mathrm{MHz}, \mathrm{CDCl}_{3}\right): \delta 9.79(\mathrm{~s}, 1 \mathrm{H}), 7.40(\mathrm{~m}, 2 \mathrm{H}), 6.99(\mathrm{~d}, J=7.7 \mathrm{~Hz}$, $1 \mathrm{H}), 4.22(\mathrm{t}, J=4.2 \mathrm{~Hz}, 2 \mathrm{H}), 3.86(\mathrm{t}, J=4.2 \mathrm{~Hz}, 2 \mathrm{H}), 3.72(\mathrm{~m}, 2 \mathrm{H})$, 3.68-3.63 (m, 36H), 3.53 (t, $J=4.2 \mathrm{~Hz}, 2 \mathrm{H}$ ), 3.37 (s, 3H), 3.09 (bs, $1 \mathrm{H}) ;{ }^{13} \mathrm{C} \mathrm{NMR}(100.62 \mathrm{MHz}, \mathrm{CDCl} 3)$ : $\delta 190.7,153.6,146.8,129.2$, $127.9,115.7,112.1,71.8,70.5,70.4,70.3,70.2,69.2,68.8,59.0$.

Compound (16): In a $100 \mathrm{~mL}$ round-bottom flask equipped with a septum and a magnetic stirrer under nitrogen atmosphere, 15 $(2.40 \mathrm{~g}, 3.77 \mathrm{mmol})$ was dissolved in DMF $(28 \mathrm{~mL})$. Potassium carbonate $(625.0 \mathrm{mg}, 4.52 \mathrm{mmol})$ and 1,2-dibromoethane (1.3 $\mathrm{mL}, 15.1 \mathrm{mmol}$ ) were added and the resulting yellow suspension was stirred at $60{ }^{\circ} \mathrm{C}$ overnight. Upon completion, the reaction mixture was concentrated in vacuo and the residue was dissolved in $\mathrm{CH}_{2} \mathrm{Cl}_{2}(50 \mathrm{~mL})$ and $\mathrm{H}_{2} \mathrm{O}(20 \mathrm{~mL})$. The aqueous layer was separated and extracted with $\mathrm{CH}_{2} \mathrm{Cl}_{2}(3 \times 20 \mathrm{~mL})$. The combined organic layers were washed with brine, dried over anhydrous $\mathrm{MgSO}_{4}$ and concentrated in vacuo. The resulting brownish oil was purified by column chromatography on silica gel $\left(\mathrm{CH}_{2} \mathrm{Cl}_{2} / \mathrm{MeOH}\right)$ to afford 16 as a yellowish oil (1.57 g, 56\%). $\mathrm{R}_{f} 0.53$ $\left(\mathrm{CH}_{2} \mathrm{Cl}_{2} / \mathrm{MeOH}: 9 / 1\right)$; MS (ESI positive mode): $\mathrm{m} / \mathrm{z} 745.3[\mathrm{M}+\mathrm{H}]^{+}$; IR (neat): 3579, 2867, 1686, 1595, 1586, 1509, 1435, 1348, 1266, 1096, 947, $849 \mathrm{~cm}^{-1}$; HRMS (ESI positive mode) calcd. for $\mathrm{C}_{32} \mathrm{H}_{56} \mathrm{BrO}_{14}$ : 743.284795; found: 743.284780; ${ }^{1} \mathrm{H}$ NMR (400.13 $\left.\mathrm{MHz}, \mathrm{CDCl}_{3}\right): \delta 9.83(\mathrm{~s}, 1 \mathrm{H}), 7.45(\mathrm{~m}, 2 \mathrm{H}), 6.98(\mathrm{~d}, J=8.8 \mathrm{~Hz}$, $1 \mathrm{H}), 4.39(\mathrm{t}, J=6.4 \mathrm{~Hz}, 2 \mathrm{H}), 4.22(\mathrm{t}, J=4.7 \mathrm{~Hz}, 2 \mathrm{H}), 3.89(\mathrm{t}, J=$ $4.4 \mathrm{~Hz}, 2 \mathrm{H}), 3.75(\mathrm{~m}, 2 \mathrm{H}), 3.68(\mathrm{t}, J=6.4 \mathrm{~Hz}, 2 \mathrm{H}), 3.62(\mathrm{~m}, 36 \mathrm{H})$, $3.53(\mathrm{~m}, 2 \mathrm{H}), 3.36(\mathrm{~s}, 3 \mathrm{H}) ;{ }^{13} \mathrm{C}$ NMR $\left(100.62 \mathrm{MHz}, \mathrm{CDCl}_{3}\right): \delta$ 190.7, 153.3, 149.3, 130.8, 126.3, 113.4, 112.2, 71.8, 70.9, 70.6, $70.5,70.4,69.4,68.9,58.9,28.6$.

Compound (17): In a $25 \mathrm{~mL}$ round-bottom flask equipped with a septum and a magnetic stirrer under nitrogen atmosphere, 16 (775.0 mg, $1.04 \mathrm{mmol}$ ) was dissolved in THF $(2.5 \mathrm{~mL})$ and the reaction mixture was cooled to $0{ }^{\circ} \mathrm{C}$. Subsequently, sodium borohydride $(39.4 \mathrm{mg}, 1.04 \mathrm{mmol})$ and $\mathrm{MeOH}(0.250 \mathrm{~mL})$ were added slowly. The resulting colorless solution was stirred at $0{ }^{\circ} \mathrm{C}$ for 30 min until complete disappearance of starting material (monitored by TLC). Upon completion, the reaction mixture was quenched with a $1 \mathrm{M} \mathrm{HCl}$ aqueous solution and concentrated in vacuo. The residue was dissolved in $\mathrm{CH}_{2} \mathrm{Cl}_{2}(20 \mathrm{~mL})$ and $\mathrm{H}_{2} \mathrm{O}(10$ $\mathrm{mL})$. The aqueous layer was separated and extracted with $\mathrm{CH}_{2} \mathrm{Cl}_{2}$ $(3 \times 10 \mathrm{~mL})$. The combined organic layers were washed with brine, dried over anhydrous $\mathrm{MgSO}_{4}$ and concentrated in vacuo to afford 17 as a colorless oil ( $660.5 \mathrm{mg}, 85 \%)$. The crude product was engaged in the next step without further purification. $\mathrm{R}_{f} 0.48$ $\left(\mathrm{CH}_{2} \mathrm{Cl}_{2} / \mathrm{MeOH}\right.$ : 9/1); MS (ESI positive mode): $\mathrm{m} / \mathrm{z} 762.3$ $\left[\mathrm{M}+\mathrm{NH}_{4}\right]^{+}$; IR (neat): 3467, 2867, 1607, 1591, 1510, 1455, 1427, 1349, 1262, 1097, 949, 851, $568 \mathrm{~cm}^{-1}$; HRMS (ESI positive mode) calcd. for $\mathrm{C}_{32} \mathrm{H}_{61} \mathrm{BrNO}_{14}$ : 762.326994; found: 762.327166; ${ }^{1} \mathrm{H}$ $\operatorname{NMR}\left(400.13 \mathrm{MHz}, \mathrm{CDCl}_{3}\right): \delta 6.94(\mathrm{~s}, 1 \mathrm{H}), 6.82(\mathrm{~m}, 2 \mathrm{H}), 4.52(\mathrm{~s}$, $2 \mathrm{H}$ ), 4.24 (t, $J=6.4 \mathrm{~Hz}, 2 \mathrm{H}), 4.12(\mathrm{t}, J=4.8 \mathrm{~Hz}, 2 \mathrm{H}), 3.80$ (t, $J=$ $4.9 \mathrm{~Hz}, 2 \mathrm{H}), 3.67(\mathrm{~m}, 2 \mathrm{H}), 3.61-3.54(\mathrm{~m}, 38 \mathrm{H}), 3.49(\mathrm{~m}, 2 \mathrm{H}), 3.31$ (s, 3H), 2.75 (bs, $1 \mathrm{H}) ;{ }^{13} \mathrm{C}$ NMR (100.62 MHz, $\mathrm{CDCl}_{3}$ ): $\delta 149.1$, 147.0, 135.8, 119.7, 116.0, 113.5, 71.7, 70.6, 70.4, 70.3, 69.7, $69.5,68.6,64.3,58.8,29.4$.

Compound (18): In a $25 \mathrm{~mL}$ round-bottom flask equipped with a septum and a magnetic stirrer under nitrogen atmosphere, 17 (600.0 mg, $0.81 \mathrm{mmol})$ was dissolved in THF $(2 \mathrm{~mL})$. Then, 3,4dihydro-2H-pyran (147 $\mu \mathrm{L}, 1.62 \mathrm{mmol})$ and pyridinium $\mathrm{p}$ toluenesulfonate $(20.4 \mathrm{mg}, 0.08 \mathrm{mmol})$ previously dissolved in $\mathrm{CH}_{2} \mathrm{Cl}_{2}(0.4 \mathrm{~mL})$ were added. The reaction mixture was stirred at room temperature overnight until complete disappearance of starting material. Subsequently, the crude was concentrated in vacuo and the residue was dissolved in $\mathrm{CH}_{2} \mathrm{Cl}_{2}(20 \mathrm{~mL})$ and $\mathrm{H}_{2} \mathrm{O}$ $(10 \mathrm{~mL})$. The aqueous layer was separated and extracted with $\mathrm{CH}_{2} \mathrm{Cl}_{2}(3 \times 10 \mathrm{~mL})$. The combined organic layers were washed with brine, dried over anhydrous $\mathrm{MgSO}_{4}$ and concentrated in vacuo. The resulting yellowish oil was purified by column chromatography on silica gel $\left(\mathrm{CH}_{2} \mathrm{Cl}_{2} / \mathrm{MeOH}\right)$ to afford $\mathbf{1 8}$ as a colorless oil (601.6 mg, 90\%). $\mathrm{R}_{f} 0.55\left(\mathrm{CH}_{2} \mathrm{Cl}_{2} / \mathrm{MeOH}: 9 / 1\right)$; $\mathrm{MS}$ (ESI positive mode): $\mathrm{m} / \mathrm{z} 846.4\left[\mathrm{M}+\mathrm{NH}_{4}\right]^{+}$; IR (neat): 2867,1511 , 1455, 1349, 1264, 1099, 1033, 949, 868, $852 \mathrm{~cm}^{-1}$; HRMS (ESI positive mode) calcd. for $\mathrm{C}_{37} \mathrm{H}_{69} \mathrm{BrNO}_{15}$ : 846.384509; found: 846.384788; ${ }^{1} \mathrm{H}$ NMR (400.13 MHz, $\left.\mathrm{CDCl}_{3}\right): \delta 6.87$ (s, $\left.1 \mathrm{H}\right), 6.81$ (s, 1H), $4.60(\mathrm{~m}, 2 \mathrm{H}), 4.33(\mathrm{~d}, J=11.7 \mathrm{~Hz}, 1 \mathrm{H}), 4.22(\mathrm{t}, J=4.8$ $\mathrm{Hz}, 2 \mathrm{H}), 4.09$ (t, $J=4.8 \mathrm{~Hz}, 2 \mathrm{H}), 3.82(\mathrm{~m}, 1 \mathrm{H}), 3.79(\mathrm{t}, J=4.8 \mathrm{~Hz}$, $2 \mathrm{H}), 3.66(\mathrm{~m}, 2 \mathrm{H}), 3.58-3.54(\mathrm{~m}, 40 \mathrm{H}), 3.46(\mathrm{t}, J=4.8 \mathrm{~Hz}, 2 \mathrm{H})$, $3,29(\mathrm{~s}, 3 \mathrm{H}), 1.79-1.44(\mathrm{~m}, 6 \mathrm{H}) ;{ }^{13} \mathrm{C}$ NMR $\left(100.62 \mathrm{MHz}, \mathrm{CDCl}_{3}\right): \delta$ 149.0, 147.2, 132.4, 120.8, 116.0, 114.3, 97.3, 71.6, 70.5, 70.4, $70.3,70.2,69.6,69.4,68.6,68.2,61.9,58.7,30.2,29.3,25.2$, 19.3 .

Compound (19): In a $25 \mathrm{~mL}$ round-bottom flask equipped with a septum and a magnetic stirrer under nitrogen atmosphere, 2 (20.0 $\mathrm{mg}, 0.045 \mathrm{mmol}$ ) and cesium carbonate $(58.6 \mathrm{mg}, 0.180 \mathrm{mmol}$ ) were dissolved in DMF $(7 \mathrm{~mL})$. The pale yellow suspension was stirred at $80^{\circ} \mathrm{C}$ for $15 \mathrm{~min}$. Then, 18 (130.7 $\left.\mathrm{mg}, 0.158 \mathrm{mmol}\right)$ previously dissolved in DMF $(1 \mathrm{~mL})$, was added dropwise and the reaction mixture was stirred at $80{ }^{\circ} \mathrm{C}$ for 24 hours. Upon completion, the mixture was allowed to cool to room temperature and concentrated in vacuo. The resulting residue was taken up in $\mathrm{CH}_{2} \mathrm{Cl}_{2}$, filtered through a pad of Celite ${ }^{\circledR}$ and the filtrate was concentrated in vacuo to afford a brownish oil. The crude was 
purified by preparative TLC $\left(\mathrm{CH}_{2} \mathrm{Cl}_{2} / \mathrm{MeOH} 95: 5\right)$ and the expected compound 19 was obtained as a brownish oil $(90.9 \mathrm{mg}$, 74\%). $\mathrm{R}_{f}$ 0.41( $\left.\mathrm{CH}_{2} \mathrm{Cl}_{2} / \mathrm{MeOH}: 9 / 1\right)$; $\mathrm{MS}$ (ESI positive mode): $\mathrm{m} / \mathrm{z}$ $1388.9([\mathrm{M}+2 \mathrm{Na}] / 2+21)$; IR (neat): 3516, 2867, 1715, 1640, $1510,1454,1349,1252,1098,1034,948,851,814,536 \mathrm{~cm}^{-1} .{ }^{1} \mathrm{H}$ NMR (400.13 MHz, $\left.\mathrm{CDCl}_{3}\right): \delta 7.71(\mathrm{~s}, 1 \mathrm{H}), 7.27-7.20(\mathrm{~m}, 2 \mathrm{H})$, 7.03-6.85 (m, 12H), 6.69-6.54 (m, 2H), 4.73-4.58 (m, 9H), $4.41(\mathrm{~d}$, $J=11.9 \mathrm{~Hz}, 3 \mathrm{H}), 4.36-4.15(\mathrm{~m}, 12 \mathrm{H}), 3.90-3.80(\mathrm{~m}, 9 \mathrm{H}), 3.63$ $3.54(\mathrm{~m}, 120 \mathrm{H}), 3.37(\mathrm{~s}, 9 \mathrm{H}), 1.86-1.52(\mathrm{~m}, 18 \mathrm{H}) ;{ }^{13} \mathrm{C} \mathrm{NMR}$ $\left(100.62 \mathrm{MHz}, \mathrm{CDCl}_{3}\right): \delta 157.5,157.4,149.1,148.2,141.6,141.1$, $140.6,140.4,134.6,134.1,132.2,132.1,131.9,131.4,131.3$, $130.9,121.5,121.3,121.0,116.3,115.5,115.3,115.1,114.8$, $114.5,114.3,113.0,97.7,93.4,84.6,71.9,71.5,70.6,69.7,69.6$, $68.8,68.7,68.2,66.5,62.3,59.1,36.5,36.2,30.6,29.8,25.5$, 19.5 .

Compound (20): 2-((4-(2-bromoethoxy)-3-methoxy benzyl)oxy)tetrahydro-2H-pyran (465 mg, $1.35 \mathrm{mmol})$ was added to a stirred solution of $2(100 \mathrm{mg}, 0.23 \mathrm{mmol})$ and cesium carbonate $(440 \mathrm{mg}, 1.35 \mathrm{mmol})$ in dry DMF $(15 \mathrm{~mL})$. The solution was heated for 3 days at $80^{\circ} \mathrm{C}$ under nitrogen. The solvent was stripped off and the residue was extracted with dichloromethane / water. The combined organic layers were washed with brine, dried over $\mathrm{MgSO}_{4}$ and evaporated to leave an oily residue. A purification by chromatography on silica gel (cyclohexane/EtOAc 100:0 to 70:30) afforded product 20 as a pale yellow solid (207 $\mathrm{mg}, 73 \%$ ). $\mathrm{R}_{f} 0.48$ (Cyclohexane/EtOAc 8/2); MS (ESI positive mode): $\mathrm{m} / \mathrm{z} 846.4$ [M+NH4]+; IR (neat): 2944, 1609, 1516, 1478, 1234, 1136, 1026, 794, 741, $549 \mathrm{~cm}^{-1}$; HRMS (ESI positive mode) calcd. for $\mathrm{C}_{66} \mathrm{H}_{77} \mathrm{INaO}_{15}$ : 1259.419940 ; found: $1259.419582 .{ }^{1} \mathrm{H}$ $\operatorname{NMR}\left(400.13 \mathrm{MHz}, \mathrm{CDC}_{\mathrm{l}}\right)$ : $\delta 7.72(\mathrm{~s}, 1 \mathrm{H}), 7.30-7.24(\mathrm{~m}, 1 \mathrm{H}), 7.21$ $(\mathrm{d}, J=8.4 \mathrm{~Hz}, 1 \mathrm{H}), 6.99(\mathrm{~d}, J=8.0 \mathrm{~Hz}, 1 \mathrm{H}), 6.96-6.85(\mathrm{~m}, 11 \mathrm{H})$, 6.69 (dd, $J=8.5 \mathrm{~Hz}, J=2.5 \mathrm{~Hz}, 1 \mathrm{H}$ ), 6.64 (dd, $J=8.5 \mathrm{~Hz}, J=2.5$ $\mathrm{Hz}, 1 \mathrm{H}), 4.77-4.63(\mathrm{~m}, 9 \mathrm{H}), 4.44(\mathrm{~d}, J=11.8 \mathrm{~Hz}, 3 \mathrm{H}), 4.41-4.21$ $(\mathrm{m}, 12 \mathrm{H}), 3.97-3.89(\mathrm{~m}, 3 \mathrm{H}), 3.88(\mathrm{~s}, 3 \mathrm{H}), 3.86(\mathrm{~s}, 3 \mathrm{H}), 3.85(\mathrm{~s}$, $3 \mathrm{H})$, 3.66-3.58 (m, 3H), 3.58-3.50 (m, 3H), 1.92-1.79 (m, $3 \mathrm{H})$, $1.78-1.68(\mathrm{~m}, 3 \mathrm{H}), 1.68-1.47(\mathrm{~m}, 12 \mathrm{H}) ;{ }^{13} \mathrm{C}$ NMR $(100.62 \mathrm{MHz}$, $\left.\mathrm{CDCl}_{3}\right): \delta 157.3,156.0,149.5,147.4,141.4,140.9,140.4,140.2$, $134.0,131.6,131.2,131.0,130.6,120.5,120.4,116.2,114.3$, $114.0,113.8,113.8,112.9,112.8,113.0,111.9,97.5,84.5,68.6$, $68.1,67.6,66.2,62.2,55.9,55.8,36.3,36.0,30.5,25.3,19.4$.

Compound (21): In a $50 \mathrm{~mL}$ round-bottom flask equipped with a septum and a magnetic stirrer, $19(40.0 \mathrm{mg}, 0.015 \mathrm{mmol})$ was dissolved in formic acid $(20 \mathrm{~mL})$. The reaction mixture was slowly stirred at $55^{\circ} \mathrm{C}$ for 3 hours. Subsequently, the colorless solution was allowed to cool to room temperature and concentrated in vacuo. The brownish crude oil was purified by preparative HPLC (water/acetonitrile 100:0 to 0:100) to afford 21 as a yellow oil (14.5 mg, 41\%). $\mathrm{R}_{f} 0.50\left(\mathrm{CH}_{2} \mathrm{Cl}_{2} / \mathrm{MeOH}: 9 / 1\right)$; MS (ESI positive mode): $\mathrm{m} / \mathrm{z} 1347.6 \pm 22,[(\mathrm{M}+2 \mathrm{H}) / 2]^{+}$; IR (neat): 3062, 3031, 2981, 2925, 2867, 2231, 1603, 1496, 1454, 1286, 1246, 1021, 966, 732, 695, $646 \mathrm{~cm}^{-1} .{ }^{1} \mathrm{H}$ NMR $\left(400.13 \mathrm{MHz}, \mathrm{CDC}_{13}\right): \delta 7.21-6.39(\mathrm{~m}, 12 \mathrm{H})$, 4.64-4.47 (m, 3H), 4.44-4.03 (m, 12H), 3.97-3.41 (m, 141H), 3.37 (s, 9H). For this compound, the ${ }^{1} \mathrm{H}$ NMR spectrum interpretation is extremely difficult due to the presence of two forms (syn/anti) of the cryptophane (confirmed by the synthesis of 22) and 3 PEG11 substituents (All attempts of separation of this two forms have not been successful). Nevertheless, the water solubility of this compound and the presence of syn and anti forms of this cryptophane have been demonstrated by the ${ }^{129} \mathrm{Xe}$ NMR spectrum included in the Supporting Information.

Compound (22)-anti: In a $250 \mathrm{~mL}$ round-bottom flask equipped with a septum and a magnetic stirrer, $20(105 \mathrm{mg}, 0.085 \mathrm{mmol})$ was dissolved in formic acid $(100 \mathrm{~mL})$. The reaction mixture was slowly stirred at $55^{\circ} \mathrm{C}$ for 5 hours and kept stirring in the room temperature for $18 \mathrm{~h}$. Subsequently, the colorless solution was cooled to room temperature and concentrated in vacuo. The crude product was purified by chromatography on silica gel (cyclohexane/chloroform 100:0 to 50:50) afforded product 22-anti as a white solid (35 mg, 44\%). $\mathrm{R}_{f} 0.25\left(\mathrm{CHCl}_{3} /\right.$ EtOAc: 9/1); MS (ESI positive mode): $\mathrm{m} / \mathrm{z}$ 948.7, [M+NH$]^{+}$; IR (neat): 2231, 1005, 948, 884, 733, 700, 626, $530 \mathrm{~cm}^{-1}$; HRMS (ESI positive mode) calcd. for $\mathrm{C}_{51} \mathrm{H}_{48} \mathrm{OO}_{9}$ : 931.233758; found: 931.233085; ${ }^{1} \mathrm{H}$ NMR $\left(400.13 \mathrm{MHz}, \mathrm{CDCl}_{3}\right): \delta 7.56(\mathrm{~s}, 1 \mathrm{H}), 7.12(\mathrm{~d}, J=8.4 \mathrm{~Hz}, 1 \mathrm{H}), 7.07$ $(\mathrm{d}, J=8.4 \mathrm{~Hz}, 1 \mathrm{H}), 6.86(\mathrm{~s}, 1 \mathrm{H}), 6.82(\mathrm{~s}, 1 \mathrm{H}), 6.79-6.72(\mathrm{~m}, 2 \mathrm{H})$, $6.70(\mathrm{~s}, 1 \mathrm{H}), 6.66(\mathrm{~s}, 3 \mathrm{H}), 6.62(\mathrm{~s}, 1 \mathrm{H}), 6.46-6.34(\mathrm{~m}, 2 \mathrm{H}), 4.69-$ $4.46(\mathrm{~m}, 6 \mathrm{H}), 4.44(\mathrm{t}, J=9.4 \mathrm{~Hz}, 2 \mathrm{H}), 4.42-4.04(\mathrm{~m}, 10 \mathrm{H}), 3.99-$ $3.89(\mathrm{~m}, 2 \mathrm{H}), 3.85(\mathrm{~s}, 3 \mathrm{H}), 3.83(\mathrm{~s}, 3 \mathrm{H}), 3.78(\mathrm{~s}, 3 \mathrm{H}), 3.57-3.36$ $(\mathrm{m}, 6 \mathrm{H}) ;{ }^{13} \mathrm{C}$ NMR $\left(100.62 \mathrm{MHz}, \mathrm{CDCl}_{3}\right): \delta 157.4,156.5,156.3$, $148.7,148.4,147.2,147.0,146.5,141.8,141.0,140.3,139.9$, 136.2, 134.3, 132.9, 132.7, 132.2, 131.5, 130.8, 130.6, 122.0, $120.1,119.1,119.0,116.6,116.5,114.8,114.0,113.1,112.0$, $111.8,87.7,77.7,66.6,65.4,65.1,56.7,56.5,55.9,36.3,36.1$, 35.9 .

Compound (22)-syn: In a $250 \mathrm{~mL}$ round-bottom flask equipped with a septum and a magnetic stirrer, $20(105 \mathrm{mg}, 0.085 \mathrm{mmol})$ was dissolved in formic acid $(100 \mathrm{~mL})$. The reaction mixture was slowly stirred at $55{ }^{\circ} \mathrm{C}$ for 5 hours and kept stirring in the room temperature for $18 \mathrm{~h}$. Subsequently, the colorless solution was cooled to room temperature and concentrated in vacuo. The crude product was purified by chromatography on silica gel (cyclohexane/chloroform 100:0 to 50:50). Then the fraction containing targeted product was purified by preparative TLC (chloroform/EtOAc 90:10) to afford the expected compound 22syn as a white solid (2 mg, 2\%). $\mathrm{R}_{f} 0.44\left(\mathrm{CHCl}_{3} / \mathrm{EtOAc:} 9 / 1\right)$; MS (ESI positive mode): $\mathrm{m} / \mathrm{z}$ 948.7, $\left[\mathrm{M}+\mathrm{NH}_{4}\right]^{+}$; IR (neat): 2231, 1005, 948, 884, 733, 700, 626, $530 \mathrm{~cm}^{-1}$; HRMS (ESI positive mode) calcd. for $\mathrm{C}_{51} \mathrm{H}_{48} \mathrm{IO}_{9}$ : 931.233758; found: 931.233400; ${ }^{1} \mathrm{H}$ NMR $\left(400.13 \mathrm{MHz}, \mathrm{CDCl}_{3}\right)$ : $\delta 7.60$ (s, $\left.1 \mathrm{H}\right), 7.13(\mathrm{~d}, J=8.6 \mathrm{~Hz}, 1 \mathrm{H}), 7.07$ $(\mathrm{d}, J=8.6 \mathrm{~Hz}, 1 \mathrm{H}), 6.87(\mathrm{~s}, 1 \mathrm{H}), 6.80(\mathrm{~s}, 1 \mathrm{H}), 6.77(\mathrm{~d}, J=2.4 \mathrm{~Hz}$, $1 \mathrm{H}), 6.76-6.64(\mathrm{~m}, 6 \mathrm{H}), 6.61(\mathrm{~d}, J=2.4 \mathrm{~Hz}, 1 \mathrm{H}), 4.69-4.46(\mathrm{~m}$, $6 \mathrm{H}), 4.45-4.17(\mathrm{~m}, 7 \mathrm{H}), 4.15-4.06(\mathrm{~m}, 1 \mathrm{H}), 4.05-3.91(\mathrm{~m}, 2 \mathrm{H}), 3.85$ $(\mathrm{s}, 3 \mathrm{H}), 3.82(\mathrm{~s}, 3 \mathrm{H}), 3.80(\mathrm{~s}, 3 \mathrm{H}), 3.76-3.68(\mathrm{~m}, 1 \mathrm{H}), 3.61-3.53$ $(\mathrm{m}, 1 \mathrm{H}), 3.52-3.37(\mathrm{~m}, 6 \mathrm{H}) ;{ }^{13} \mathrm{C}$ NMR $\left(100.62 \mathrm{MHz}, \mathrm{CDCl}_{3}\right): \delta$ $157.7,157.0,156.6,150.1,149.5,149.3,147.2,146.3,146.2$, $141.7,140.7,140.0,140.0,137.6,134.7,134.3,134.0,132.2$, $132.1,131.5,131.3,130.8,130.7,128.8,121.8,121.5,121.3$, $119.3,118.9,118.6,114.2,114.0,113.6,112.8,88.1,71.4,71.2$, $70.7,69.2,68.1,65.8,56.1,38.7,36.2,30.9,30.4,29.7,28.9$, 23.7, 23.0, 14.1, 11.0. 


\section{Acknowledgements}

B. R. and G. P. thank CEA, INSERM and plan cancer for financial support. The authors thank David Buisson, Céline Chollet, Elodie Marcon and Dr. Francois Fenaille for analysis and HPLC purification. G. P. thanks Dr. Thierry Brotin and Dr. Christophe Dugave for useful discussion.

Keywords: Cryptophane $\cdot C_{1}$-symmetry $\cdot$ Host-guest systems $\bullet$ Xenon $\cdot$ NMR spectroscopy

[1] a) M. J. Hardie, Chem. Soc. Rev. 2010, 39, 516-527; b) A. Collet, Tetrahedron, 1987, 43, 5725-5759.

[2] a) M.-Y. Ku, S.-J. Huang, S.-L. Huang, Y.-H. Liu, C.-C. Lai, S.-M. Peng, S.-H. Chiu, Chem. Commun. 2014, 50, 11709-11712; b) L.-J. Feng, H. Li, Q. Chen, B.-H. Han, RSC Adv. 2013, 3, 6985-6990; c) L. Wang, G.-T. Wang, X. Zhao, X.-K. Jiang, Z.-T. Li, J. Org. Chem. 2011, 76, 3531-3535; d) E. Huerta, H. Isla, E. M. Pérez, C. Bo, N. Martin, J. de Mendoza, J. Am. Chem. Soc. 2010, 132, 5351-5353; e) I. V. Lijanova, F. Maturano, J. G. Dominguez Chavez, K. E. Sanchez Montes, S. Hernandez Ortega, T. Klimova, M. Martinez-Garcia, Supramol. Chem. 2009, 21, 24-34; f) E. Huerta, G. A. Metselaar, A. Fragoso, E. Santos, C. Bo, J. de Mendoza, Angew. Chem. Int. Ed. 2007, 46, 202-205; g) A. M. Bond, W. Miao, C. L. Raston, T. J. Ness, M. J. Barnes, J. L. Atwood, J. Phys. Chem. B. 2001, 105, 1687-1695

[3] a) H. Zimmermann, V. Bader, R. Poupko, E. J. Wachtel, Z. Luz, J. Am. Chem. Soc. 2002, 124, 15286-15301; b) R. Lunkwitz, C. Tschierske, S. Diele, J. Mater. Chem. 1997, 7, 2001-2011.

[4] a) A. Westcott, C. J. Sumby, R. D. Walshaw, M. J. Hardie, New. J. Chem. 2009, 33, 902-912; b) D. Bardelang, F. Camerel, R. Ziessel, M. Schmutz, M. J. Hannon, J. Mater. Chem. 2008, 18, 489-494.

[5] S. Zhang, L. Echegoyen, J. Am. Chem. Soc. 2005, 127, 2006-2011.

[6] a) J. J. Henkelis, M. J. Hardie, Chem. Commun. 2015, 51, 11929-11943; b) F. L. Thorp-Greenwood, T. K. Ronson, M. J. Hardie, Chem. Sci. 2015, 6, 5779-5792; c) J. J. Henkelis, M. J. Hardie, CrystEngComm. 2014, 16, 8138-8146; d) T. K. Ronson, H. Nowell, A. Westcott, M. J. Hardie, Chem. Commun. 2011, 47, 176-178; e) C. Carruthers, J. Fisher, L. P. Harding M. J. Hardie, Dalton Trans. 2010, 39, 355-357; f) T. K. Ronson, J. Fisher L. P. Harding, P. J. Rizkallah, J. E. Warren, M. J. Hardie, Nature Chem. 2009, 1, 212-216; g) A. Westcott, J. Fisher, L. P. Harding, P. Rizkallah M. J. Hardie, J. Am. Chem. Soc. 2008, 130, 2950-2951.

[7] a) L. Erieau-Peyrard, C. Coiffier, P. Bordat, D. Bégué, S. Chierici, S. Pinet, I. Gosse, I. Baraille, R. Brown, Phys. Chem. Chem. Phys. 2015 17, 4168-4174; b) S. R. S. Sarsah, M. R. Lutz, M. Zeller, D. S. Crumrine D. P. Becker, J. Org. Chem. 2013, 78, 2051-2058; c) L. Peyrard, M.-L. Dumartin, S. Chierici, S ; Pinet, G. Jonusauskas, P. Meyrand, I. Gosse, J. Org. Chem. 2012, 77, 7023-7027; d) M.-L. Dumartin, C. Givelet, P. Meyrand, B. Bibal, I. Gosse, Org. Biomol. Chem. 2009, 7, 2725-2728.

[8] T. Brotin, J.-P. Dutasta, Chem. Rev. 2009, 88-130 and references therein

[9] K. Bartik, M. Luhmer, J.-P. Dutasta, A. Collet, J. Reisse, J. Am. Chem. Soc. 1998, 120, 784-791.

[10] a) B. A. Riggle, Y. Wang, I. J. Dmochowski, J. Am. Chem. Soc. 2015 , 137, 5542-5548; b) C. Witte, V. Martos, H. M. Rose, S. Reinke, S. Klippel, L. Schröder, C. P. R. Hackenberger, Angew. Chem. Int. Ed. 2015, 54, 2806-2810; c) N. S. Khan, B. A. Riggle, G. Seward, Y. Bai, I. J. Dmochowski, Bioconjugate Chem. 2015, 26, 101-109; d) H. M. Rose, C. Witte, F. Rossella, S. Klippel, C. Freund, L. Schröder, Proc. Natl. Acad. Sci. U.S.A. 2014, 111, 11697-11702; e) N. Kotera, N. Tassali, E. Léonce, C. Boutin, P. Berthault, T. Brotin, J.-P. Dutasta, L. Delacour, T. Traoré, D.-A. Buisson, F. Taran, S. Coudert, B. Rousseau, Angew. Chem. Int. Ed. 2012, 51, 4100-4103; f) T. K. Stevens, K. K. Palaniappan, R. M. Ramirez, M. B. Francis, D. E. Wemmer, A. Pines, Magn. Reson. Med. 2013, 69, 1245-1252; g) G. K. Seward, Y. Bai, N. S. Khan, I. J. Dmochowski, Chem. Sci. 2011, 2, 1103-1110; h) T. Meldrum, K. L. Seim,
V. S. Bajaj, K. K. Palaniappan, W. Wu, M. B. Francis, D. E. Wemmer, A Pines, J. Am. Chem. Soc. 2010, 132, 5936-5937; i) V. Roy, T. Brotin, J. P. Dutasta, M.-H. Charles, T. Delair, F. Mallet, G. Huber, H. Desvaux, Y. Boulard, P. Berthault, ChemPhysChem. 2007, 8, 2082-2085; j) L. Schröder, T. J. Lowery, C. Hilty, D. E. Wemmer, A. Pines, Science, 2006, 314, 446-449; k) C. Hilty, T. J. Lowery, D. Wemmer, A. Pines, Angew. Chem. Int. Ed. 2006, 45, 70-73; I) T. J. Lowery, S. Garcia, L. Chavez, E. J. Ruiz, T. Wu, T. Brotin, J.-P. Dutasta, D. S. King, P. G. Schultz, A. Pines, D. E. Wemmer, ChemBioChem 2006, 7, 65-73; m) M. M. Spence, E. J. Ruiz, S. M. Rubin, T. J. Lowery, N. Winssinger, P. G. Schultz, D. E. Wemmer, A. Pines, J. Am. Chem. Soc. 2004, 126, 15287-15294.

[11] a) J.-R. Song, Z.-T. Huang, Q.-Y. Zheng, Tetrahedron 2013, 69, 73087313; b) A. Chakrabarti, H. M. Chawla, G. Hundal, N. Pant, Tetrahedron 2005, 61, 12323-12329; c) A. S. Lindsey, J. Chem. Soc. 1965, 1685 1692; d) M. M. Spence, S. M. Rubin, I. E. Dimitrov, E. J. Ruiz, D; E. Wemmer, A. Pines, S. Q. Yao, F. Tian, P. G. Schultz, Proc. Natl. Acad. Sci. U.S.A. 2001, 98, 10654-10657; e) M. M. Spence, E. J. Ruiz, S. M. Rubin, T. J. Lowery, N. Winssinger, P. G. Schultz, D. E. Wemmer, A. Pines, J. Am. Chem. Soc. 2004, 126, 15287-15294.

[12] a) T. Traoré, L. Delacour, N. Kotera, G. Merer, D.-A. Buisson, C. Dupont, B. Rousseau, Org. Process Res. Dev. 2011, 15, 435-437; b) T. Traoré, L. Delacour, S. Garcia-Argote, P. Berthault, J.-C. Cintrat, B. Rousseau, Org. Lett. 2010, 12, 960-962

[13] a) J.-T. Yu, Z. T. Huang, Q. Y. Zheng, Org. Biomol. Chem. 2012, 10, 1359-1364; b) J.-T. Yu, J. Sun, Z.-T. Huang, Q.-Y. Zheng CrystEngComm. 2012, 14, 112-115; c) D. Xu, R. J. Warmuth, J. Am. Chem. Soc. 2008, 130, 7520-7521.

[14] N. Miyaura, A. Suzuki, Chem. Rev. 1995, 95, 2457-2483.

[15] a) E. Dubost, N. Kotera, S. Garcia-Argote, Y. Boulard, E. Léonce, C Boutin, P. Berthault, C. Dugave, B. Rousseau, Org. Lett. 2013, 15, 2866 2868; b) L. Delacour, N. Kotera, T. Traoré, S. Garcia-Argote, C. Puente F. Leteurtre, E. Gravel, N. Tassali, C. Boutin, E. Léonce, Y. Boulard, P. Berthault, B. Rousseau, Chem. Eur. J. 2013, 19, 6089-6093 ; c) T. Traoré, G. Clavé, L. Delacour, N. Kotera, P.-Y. Renard, A. Romieu, P. Berthault, C. Boutin, N. Tassali, B. Rousseau, Chem. Commun. 2011, 47, 9702 9704.

[16] C. Boutin, A. Stopin, F. Lenda, T. Brotin, J.-P. Dutasta, N. Jamin, A Sanson, Y. Boulard, F. Leteurtre, G. Huber, A. Bogaert-Buchmann, N Tassali, H. Desvaux, M. Carrière, P. Berthault, Bioorg. Med. Chem. 2011, 19, 4135-4143.

[17] L.-L. Chapellet, J. R. Cochrane, E. Mari, C. Boutin, P. Berthault, T. Brotin, J. Org. Chem. 2015, 80, 6143-6151.

[18] S. V. Kessar, Y. P. Gupta, T. Mohammad, M. Goyal, K. K. Sawal, J. Chem. Soc., Chem. Commun. 1983, 400-401. 